\title{
DOUBLE-STEPPED ADAPTIVE CONTROL FOR HYBRID SYSTEMS WITH UNKNOWN MARKOV JUMPS AND STOCHASTIC NOISES*
}

\author{
SHUPING TAN $^{1}$ AND JI-FENG ZHANG ${ }^{2}$
}

\begin{abstract}
This paper is concerned with the sampled-data based adaptive linear quadratic (LQ) control of hybrid systems with both unmeasurable Markov jump processes and stochastic noises. By the least matching error estimation algorithm, parameter estimates are presented. By a double-step (DS) sampling approach and the certainty equivalence principle, a sampled-data based adaptive LQ control is designed. The DS-approach is characterized by a comparatively large estimation step for parameter estimation and a sufficient small control step for control updating. Under mild conditions, the closed-loop system is shown to be stable. It is found that the key factor determining the performance index is the estimation step rather than the control step. When the estimation step becomes too small, the system performance will become worse. When the estimation step is fixed, the system performance can indeed be improved by reducing the control step, but cannot reach the optimal value. The index difference between the sampled-data based adaptive LQ control and the conventional LQ optimal control is asymptotically bounded by a constant depending on the estimation step and the priori information of the parameter set.
\end{abstract}

Mathematics Subject Classification. 93E15, 93E35.

Received January 7, 2007. Revised June 9, 2008.

Published online August 20, 2008.

\section{INTRODUCTION}

Systems with Markov jump parameters belong to the category of "hybrid systems", which are emerging as a convenient mathematical framework for the formulation of various design problems such as target tracking, fault tolerant control and manufacturing processes, etc. [18]. Considering that the deterministic controllability of each subsystem $\left(A_{i}, B_{i}\right)$ does not ensure the existence of finite steady-state control for Markov jump system (MJS), Ji and Chizeck introduced the stochastic controllability and stabilizability concepts, gave sufficient and necessary conditions for continuous-time MJS, and derived the linear quadratic (LQ) optimal control in $[12,13]$. When the system state is continuously measurable, Sworder [23] solved the quadratic optimal

Keywords and phrases. Sampling control system, Markov jump parameter, adaptive control, double-step approach, stochastic noise, least matching error estimation.

* This work was done when the first author pursued her Ph.D. degree at the Academy of Mathematics and Systems Science, Chinese Academy of Sciences, and was partly supported by the National Natural Science Foundation of China under grants 90405017,60674038 .

1 National Laboratory of Space Intelligent Control, Beijing Institute of Control Engineering, P.O. Box 2729, Beijing 100190,

P. R. China. sptan@amss.ac.cn

2 Academy of Mathematics and Systems Science, Chinese Academy of Sciences, Beijing 100190, P. R. China. jif@iss.ac.cn 
regulator problem of linear MJS with stochastic noises; Dufour and Betrand [6], Caines and Zhang [3], Huang and Guo [10] considered stabilization controls of various stochastic jump systems; Xue and Guo [26] presented sufficient and necessary conditions on adaptive stabilization for discrete-time linear MJS where a distinguishable condition is appended to the general assumptions. When only output observations are available, Elliott and Krishnamurthy $[7,8]$ presented a class of finite-dimensional filters for continuous-time stochastic systems with time-varying parameters, and an expectation maximization algorithm to get maximum likelihood estimates of the unknown parameters involved.

We consider the sampled-data (SD) based adaptive LQ control of MJS with unknown jump parameters and stochastic noise:

$$
\mathrm{d} x_{t}=A\left(\theta_{t}\right) x_{t} \mathrm{~d} t+B\left(\theta_{t}\right) u_{t} \mathrm{~d} t+C\left(\theta_{t}\right) \mathrm{d} W_{t},
$$

where $x_{t} \in \mathbb{R}^{n}, u_{t} \in \mathbb{R}^{m}$ are system state and input, respectively; $\left\{W_{t}, \mathcal{F}_{t}^{W}\right\}$ is normal Brownian motion defined on probability space $(\Omega, \mathcal{F}, P)$ with $\mathcal{F}_{t}^{W}=\sigma\left(W_{s}, s \leq t\right) ;\left\{\theta_{t}, \mathcal{F}_{t}^{\theta}\right\}$ is a stationary Markov process taking values on a finite set $\mathcal{S}=\{1, \ldots, N\}$, where $\mathcal{F}_{t}^{\theta}=\sigma\left(\theta_{s}, s \leq t\right)$ with transition probability matrix [25]

$$
P(\tau)=\left[P_{i j}(\tau)\right]=\left[P\left(\theta_{t+\tau}=j \mid \theta_{t}=i\right)\right]=\mathrm{e}^{\Lambda \tau}, \quad \tau \geq 0 .
$$

Here $\Lambda=\left(\lambda_{i j}\right), \lambda_{i j} \geq 0, j \neq i$, and

$$
\lambda_{i i}=-\sum_{j=1, j \neq i}^{N} \lambda_{i j} .
$$

For simplicity of expression, here and hereafter we suppose the initial time $t_{0}=0$, initial state $x_{0}$ and initial parameter $\theta_{0}$ are constants; for each matrix $F\left(\theta_{t}\right)$ depending only on $\theta_{t}$, we denote $F\left(\theta_{t}\right)$ as $F_{i}$ when $\theta_{t}=i$, that is, $F_{i} \triangleq F\left(\theta_{t}\right) \mid \theta_{t}=i$. For example, denote $A\left(\theta_{t}\right) \mid \theta_{t}=i$ as $A_{i}$, and $B\left(\theta_{t}\right) \mid \theta_{t}=i$ as $B_{i}$.

In the following study of the SD-based adaptive LQ control, we have two purposes: one is to design an SD-based adaptive control to stabilize the system (1.1); and the other is to study the impact of sample size on the following quadratic performance index:

$$
J(u)=\limsup _{T \rightarrow \infty} \frac{1}{T} E\left\{\int_{0}^{T}\left(x_{t}^{T} Q\left(\theta_{t}\right) x_{t}+u_{t}^{T} R\left(\theta_{t}\right) u_{t}\right) \mathrm{d} t \mid x_{0}, \theta_{0}\right\},
$$

where $R_{i}>0, Q_{i} \geq 0$.

The idea of using digital computers as components in control systems emerged around 1950 and there was substantial development of digital computer technology in 1960s. Practically, most control systems developed today are based on computer control (SD-based control) [1]. Since 1960s, many significant results have been obtained on SD-based control, for instance, robust SD-based adaptive control [19,28], robust SD-based constrained control [9], SD-based adaptive tracking control [15,21,29], SD-based optimal control and $L_{p}$ stabilization control [14], robust SD-based stabilization analysis [20], and so on. For SD-based control design, the choice of sample step is always the primary task, since improper sample step may destroy the controllability and stability of the sampled system. Ilchmann and Townley specially discussed the methods of choosing sample step in [11]. For MJS with known parameters and stochastic noises, Yao and Zhang found that small sample step always favored the LQ performance [27]. For MJS with unknown parameters which is free of noises, Tan et al. pointed out in [24] that the following SD-based adaptive LQ control

$$
\begin{aligned}
u_{t} & =-L\left(\hat{\theta}_{k h}\right) x_{k h}, \quad t \in[k h,(k+1) h), \\
\hat{\theta}_{k h} & =\arg \min _{i \in \mathcal{S}}\left\|x_{k h}-\mathrm{e}^{\left(A_{i}-B_{i} L\left(\hat{\theta}_{(k-1) h}\right) h\right.} x_{(k-1) h}\right\|,
\end{aligned}
$$

approached to the conventional control in terms of system performance as the sample step went to zero. In other words, the smaller the sample step is, the closer the LQ performance index under the SD-based control is to the true optimal value. 
However, the systems discussed in this paper is much more intricate than the cases mentioned above, since the parameters not only obey with Markov jump process but are also unknown, besides, the system is with stochastic noises. By (1.2), when sample size is sufficiently small, the transition probability of the system jumping from subsystem $\left[A_{i}, B_{i}\right]$ to another subsystem $\left[A_{j}, B_{j}\right]$ is approximately $\lambda_{i j} h$. Hence, for linear MJS, the changes of the norm of state $x$ caused by different subsystems while jumping from the same initial state during same period, is linear with the sample size $h$. On the other hand, the standard difference of sequence $W_{k h}$ derived from sampling the Brownian motion is linear with $h^{\frac{1}{2}}$. As a consequence, when Brownian motion and unknown jumps co-exist in the systems, if the sample size is much more smaller than 1 , then the state norm difference induced by the system noise will much greater than that induced by the jumps, that is, the information used to estimate the jump parameters will be spoiled by the noise. Consequently, small sample size is not always favorable to the parameter estimation, and hence, to the performance of the SD-based adaptive control. In this case, the SD-based adaptive control is no longer asymptotically optimal as the sample size goes to zero, and reducing sample size is no longer the best strategy to improve system performance.

Based on the above analysis, considering the coupled influence of Markov jumps and Brownian motion on the estimation of system state, in order to minimize the influence of Brownian motion and optimize the LQ performance index, we introduce a double-step (DS) approach to design SD-based adaptive control. Precisely, we use two different steps to construct parameter estimation and feedback control, respectively. One step is used to estimate system parameters, denoted by $h^{*}$, called estimation step; the other is to design SD-based control, denoted by $h$, called control step. It is found that to get a good performance index, it is better to choose a comparatively large step for parameter estimation, and a sufficiently small step for control updating. Under mild conditions, the stability of the closed-loop system is proved. It is pointed out that the estimation step is the key to determining the performance index. When the estimation step is fixed, the performance index can indeed be improved by reducing the control step, but cannot reach the optimal value. The index difference between the SD-based adaptive LQ control and the conventional LQ optimal control is asymptotically bounded by a constant depending on the estimation step and the priori information of the parameter set.

The rest of this paper is organized as following. In Section 2 we design the SD-based adaptive control. In Section 3 we give our main result Theorem 3.1 and analyze how the estimation step and the control step influence the system LQ performance index. In Section 4 we give some preliminary lemmas and the proof of Theorem 3.1. In Section 5 we illustrate our result by a simulation example. A brief conclusion of this paper is given in Section 6.

\section{Design of the SD-BASED ADAPtive CONTROL}

In this paper we only discuss the following case where: (i) for each $i \in \mathcal{S}, A_{i}, B_{i}$ are known but $\theta_{t}$ is unknown; (ii) the system is with stochastic noise; and (iii) only the sampled information of the system state $x_{k h}$ are available. So, in this paper, we have new information coming in only at the sample time instant, and have only the sampled state information rather than the complete state information available for estimating the jump process $\theta_{t}$ and designing the adaptive control law.

By the condition on the jump parameter, almost all sample paths $\theta_{t}$ are constant except for a finite number of simple jumps in any given finite time interval. So, we can define the paths of $x_{t}$ in an obvious way by joining solutions arcs of (1.1) at jump points of $\theta$. The $x_{t}$ sample paths so determined are then continuous with probability one [25].

Consider the following MJS which is free of stochastic noise

$$
\mathrm{d} x_{t}=A\left(\theta_{t}\right) x_{t} \mathrm{~d} t+B\left(\theta_{t}\right) u_{t} \mathrm{~d} t
$$

where $\left\{\theta_{t}, t \geq 0\right\}$ is a stationary Markov jump process with transition probability matrix (1.2)-(1.3). 
Definition 2.1 ([12]). We say that the systems $(2.1),(1.2)-(1.3)$, or simply $\left[A\left(\theta_{t}\right), B\left(\theta_{t}\right)\right]$, is stochastically stabilizable, if for all finite $x_{0} \in \mathbb{R}^{n}$ and $\theta_{0} \in \mathcal{S}$, there exists a linear feedback control law:

$$
u_{t}=-L\left(\theta_{t}\right) x_{t}
$$

with $L\left(\theta_{t}\right)$ being constant for each value of $\theta_{0} \in \mathcal{S}$, and $\left\|L\left(\theta_{t}\right)\right\|<\infty$ such that for a symmetric positive definite matrix $\Pi$,

$$
E\left\{\int_{0}^{\infty} x_{t}^{T}\left(x_{0}, \theta_{0}, u\right) x_{t}\left(x_{0}, \theta_{0}, u\right) \mathrm{d} t \mid x_{0}, \theta_{0}\right\} \leq x_{0}^{T} \Pi x_{0}
$$

According to [12], if $\forall i \in \mathcal{S},\left[A_{i}, Q_{i}^{\frac{1}{2}}\right]$ is observable, then a necessary and sufficient condition for $\left[A\left(\theta_{t}\right), B\left(\theta_{t}\right)\right]$ to be stochastically stabilizable is that for any positive definite matrix $R_{i}$ and non-negative definite matrix $Q_{i}$, the coupled algebra Riccati equations

$$
A_{i}^{T} M_{i}+M_{i} A_{i}-M_{i} B_{i} R_{i}^{-1} B_{i}^{T} M_{i}+\sum_{j=1}^{N} \lambda_{i j} M_{j}+Q_{i}=0
$$

have a unique symmetric positive definite solution set $\left\{M_{i}, i \in \mathcal{S}\right\}$.

Remark 2.1. As pointed out in [27], when parameters $\theta_{t}$ of system (1.1) are known, the optimal and suboptimal values of the LQ performance index (1.4) respectively obtained under complete-state-information-based control and SD-based control are

and

$$
J\left(u^{*}\right)=\limsup _{t \rightarrow \infty} \frac{1}{t} E \int_{0}^{t} \operatorname{tr}\left(C^{T}\left(\theta_{s}\right) M\left(\theta_{s}\right) C\left(\theta_{s}\right)\right) \mathrm{d} s,
$$

$$
\limsup _{t \rightarrow \infty} \frac{1}{t} E \int_{0}^{t} \operatorname{tr}\left(C^{T}\left(\theta_{s}\right) M\left(\theta_{s}\right) C\left(\theta_{s}\right)\right) \mathrm{d} s+O(h),
$$

where $h$ is the sample step and $M\left(\theta_{s}\right)$ are the unique symmetric positive definite solutions of the coupled algebra Riccati equations (2.2).

In this paper we study the LQ performance problem of system (1.1) when the jump parameter $\theta_{t}$ are unknown and only the sampled state information can be employed for designing the adaptive control.

We apply the following Least Matching Error Estimation (LMEE) algorithm to estimate the unknown parameter $\theta_{t}$ at time $k h^{*}$ for each positive integer $k$ :

$$
\hat{\theta}_{k h^{*}}=\arg \min _{i \in \mathcal{S}}\left\|x_{k h^{*}}-\mathrm{e}^{\left(A_{i}-B_{i} L\left(\hat{\theta}_{(k-1) h^{*}}\right)\right) h^{*}} x_{(k-1) h^{*}}\right\|,
$$

where $h^{*}$ is the estimation step. The SD-based adaptive control law is designed as:

$$
u_{t}=-L\left(\hat{\theta}_{k h^{*}}\right) x_{k h^{*}+l h}, \quad t \in\left[k h^{*}+l h, k h^{*}+(l+1) h\right), \quad 0 \leq l \leq \frac{h^{*}}{h}-1,
$$

where $L_{i}=R_{i}^{-1} B_{i}^{T} M_{i}$, and $\left\{M_{i}, i \in \mathcal{S}\right\}$ is the unique symmetric positive definite solution set of the coupled algebra Riccati equations $(2.2) ; h$ is the sample step, also the control step, satisfying $N^{*}=\frac{h^{*}}{h} \in \mathbb{N}$. In other words, at the beginning we calculate the estimate $\hat{\theta}_{k h^{*}}$ of $\theta_{k h^{*}}$ using both the sampled data $x_{(k-1) h^{*}}, x_{k h^{*}}$, and the estimate $\hat{\theta}_{(k-1) h^{*}}$ of $\theta_{(k-1) h^{*}}$; then we design the SD-based control by using the estimate $\hat{\theta}_{k h^{*}}$ and the sampled data $x_{k h^{*}+l h}$ on time interval $\left[k h^{*},(k+1) h^{*}\right)$. To maintain the advantage of small control step as in conventional cases, meanwhile to reduce the impact of system noise on parameter estimation, we propose to choose small control step and comparatively large estimation step. It is proved in the following section that the DS-approach can greatly improve the performance index. 
We propose: firstly based on LMEE updating by estimation step, to estimate the unknown parameters on line; then by certainly equivalent principle, to design the SD-based adaptive control whose feedback matrix updating by control step; finally to analyze the stability and optimality of the closed-loop system.

\section{Performance of the Closed-Loop system}

We need the following assumptions:

Assumption 3.1. For any $i \in \mathcal{S},\left[A_{i}, Q_{i}^{\frac{1}{2}}\right]$ is observable; $\left[A\left(\theta_{t}\right), B\left(\theta_{t}\right)\right]$ is stochastically stabilizable.

Assumption 3.2. Markov process $\left\{\theta_{t}, t \geq 0\right\}$ and Brownian motion $\left\{W_{t}, t \geq 0\right\}$ are independent.

Remark 3.1. Assumptions 3.1 and 3.2 are the primary conditions of MJS when LQ optimal control problem is studied. By Assumption 3.1, the coupled algebra Riccati equations (2.2) has a unique symmetric positive definite solution set. Based on this solution set, one can design a linear feedback control law for the system (2.1) to minimize the LQ performance index (1.4).

Theorem 3.1. For MJS (1.1), denote

$$
\mathcal{D}_{i j k}\left(h^{*}\right)=\mathrm{e}^{\left(A_{i}-B_{i} L_{j}\right) h^{*}}-\mathrm{e}^{\left(A_{k}-B_{k} L_{j}\right) h^{*}}, \quad i, j, k \in \mathcal{S} .
$$

Under Assumptions 3.1 and 3.2, if the estimation step $h^{*}$ and the control step $h$ satisfy

$$
\begin{aligned}
& \operatorname{det} \mathcal{D}_{i j k}\left(h^{*}\right) \neq 0, \forall i \neq k, \\
& h^{*} \leq \min \left\{1, \frac{\sqrt{2}-1}{2 c_{1} \mathrm{e}^{c_{1}}}\right\}, \quad 5 c_{2} h^{*}\left(2 \lambda+\lambda_{1} h^{*}\right)+\frac{c_{2} \gamma_{0}}{1-\gamma_{0}}<\frac{1}{2} \\
& h \leq \min \left\{h^{*}, \frac{9-4 \sqrt{5}}{2 c_{1} \mathrm{e}^{c_{1}}}\right\}, \mathrm{e}^{c_{1} h}+2 c_{1} h \mathrm{e}^{2 c_{1} h} \leq 1+\frac{\min _{i \neq k}\left\|\mathcal{D}_{i j k}\left(h^{*}\right)\right\|}{8 c_{1}^{2} h^{* 2} \mathrm{e}^{2 c_{1} h^{*}}},
\end{aligned}
$$

then with the SD-based adaptive control (2.4), the following results are true:

(1) the closed-loop system is stable in the sense of

$$
\limsup _{t \rightarrow \infty} \frac{1}{t} E \int_{0}^{t}\left(\left\|x_{s}\right\|^{2}+\left\|u_{s}\right\|^{2}\right) \mathrm{d} s<\infty
$$

(2) the LQ performance index satisfies

$$
\begin{aligned}
\limsup _{t \rightarrow \infty} \frac{1}{t} E \int_{0}^{t}\left(x_{s}^{T} Q\left(\theta_{s}\right) x_{s}+u_{s}^{T} R\left(\theta_{s}\right) u_{s}\right) \mathrm{d} s \leq & \limsup _{t \rightarrow \infty} \frac{1}{t} E \int_{0}^{t} \operatorname{tr}\left(C^{T}\left(\theta_{s}\right) M\left(\theta_{s}\right) C\left(\theta_{s}\right)\right) \mathrm{d} s+17 \gamma_{1} c_{3}\left(\frac{2}{\gamma_{2}}+1\right)^{2} h^{*} \\
& +\frac{340 \lambda \gamma_{1} c_{2} c_{3}\left(\frac{2}{\gamma_{2}}+1\right)^{2} h^{* 2}}{1-10 c_{2} h^{*}\left(2 \lambda+\lambda_{1} h^{*}\right)-\frac{2 c_{2} \gamma_{0}}{1-\gamma_{0}}}+O\left(h^{*}\right)+O\left(h^{\frac{1}{2}}\right)
\end{aligned}
$$


where

$$
\begin{aligned}
\lambda & =(N-1)\left(\mathrm{e}^{\|\Lambda\|}-1\right), \quad \lambda_{1}=\mathrm{e}^{\|\Lambda\|}-1-\|\Lambda\|, \\
c_{1} & =\max \left\{\max _{i \in \mathcal{S}}\left\|A_{i}\right\|, \max _{i, j \in \mathcal{S}}\left\|B_{i} L_{j}\right\|\right\}, \quad c_{2}=\max _{i, j \in \mathcal{S}}\left\|M_{i} B_{i} R_{i}^{-1} B_{j}^{T} K_{j}\right\|, \\
c_{3} & =\max _{i, j \in \mathcal{S}}\left\|\left(L_{i}-L_{j}\right)^{T} R_{i}\left(L_{i}-L_{j}\right)\right\|, \\
\gamma_{0} & =2 c_{1} h^{*} \mathrm{e}^{c_{1} h^{*}}, \quad \gamma_{1}=\frac{1}{h} E \int_{0}^{h} \operatorname{tr}\left(C_{i}^{T} \mathrm{e}^{A_{i}^{T} s} \mathrm{e}^{A_{i} s} C_{i}\right) \mathrm{d} s, \\
\gamma_{2} & =\min _{i, j, k \in \mathcal{S}}\left\{\sigma_{\min }\left(\mathrm{e}^{\left(A_{k}-B_{k} L_{j}\right) h^{*}}+\mathrm{e}^{\left(A_{i}-B_{i} L_{j}\right) h^{*}}-2\left(\mathrm{e}^{A_{i} h}-\int_{0}^{h} \mathrm{e}^{A_{i}(h-\tau)} \mathrm{d} \tau B_{i} L_{j}\right)^{N^{*}}\right)\right\},
\end{aligned}
$$

and $\left\{K_{i}, i \in \mathcal{S}\right\}$ is the unique symmetric positive definite solution set of the following algebra Riccati equations

$$
\left(A_{i}-B_{i} L_{i}\right)^{T} K_{i}+K_{i}\left(A_{i}-B_{i} L_{i}\right)+\sum_{j} \lambda_{i, j} K_{j}=-I .
$$

Here $\sigma_{\min }(A)$ denotes the minimal singular value of matrix $A$.

Remark 3.2. Because $\theta_{t}$ is unknown, although we know that for any given time instant, the MJS's true dynamic is determined uniquely by one of those known subsystems, we have no idea about which is the exact one. To get a good controller, we should have the ability to distinguish the subsystem that determines the MJS's dynamic from other subsystems. Condition (3.1) is such one called distinguishable condition of the MJS, which ensures that each subsystem is distinguishable from the others.

Remark 3.3. In the case where condition (3.1) does not hold, namely, for any $h^{*}$, there exists a triple $(i, j, k)$ such that $\operatorname{det} \mathcal{D}_{i j k}\left(h^{*}\right)=0$, if $x_{k h^{*}}$ lies in the zero solution space of the equations

$$
\mathcal{D}_{i j k}\left(h^{*}\right) x=0
$$

then algorithm (2.3) has more than one solution, at least including $i$ and $k$. Hence, to ensure the uniqueness of the solution of our adaptive algorithm, we need the distinguishable condition.

For one-dimension system, condition (3.1) is equivalent to distinguishable condition [26]

$$
\left(A_{i}-A_{k}\right)-\left(B_{i}-B_{k}\right) L_{j} \neq 0
$$

For systems with a higher dimension, since

$$
\lim _{h^{*} \rightarrow 0} \frac{\mathcal{D}_{i j k}\left(h^{*}\right)}{h^{*}}=\left(A_{i}-A_{k}\right)-\left(B_{i}-B_{k}\right) L_{j},
$$

then for any MJS satisfying

$$
\operatorname{det}\left(\left(A_{i}-A_{k}\right)-\left(B_{i}-B_{k}\right) L_{j}\right) \neq 0
$$

there must exist a small $h_{0}$, such that for any $h^{*} \in\left(0, h_{0}\right]$, condition (3.1) holds. 
As an example, let us explore a two-dimension MJS (1.1)-(1.2) with $N=2$ and

$$
A_{1}=\left[\begin{array}{ll}
0 & 1 \\
0 & 0
\end{array}\right], \quad B_{1}=\left[\begin{array}{l}
0 \\
1
\end{array}\right], \quad A_{2}=\left[\begin{array}{ll}
0 & 0 \\
1 & 0
\end{array}\right], \quad B_{2}=\left[\begin{array}{l}
1 \\
0
\end{array}\right], \quad \Lambda=\left[\begin{array}{cc}
-1 & 1 \\
1 & -1
\end{array}\right],
$$

$C_{1}=C_{2}=Q_{1}=Q_{2}=R_{1}=R_{2}=I$. Then, the gain matrices of feedback control are:

$$
L_{1}=\left[\begin{array}{ll}
1 & 1.73205
\end{array}\right], \quad L_{2}=\left[\begin{array}{ll}
1.73205 & 1
\end{array}\right] .
$$

By simple calculation, it can be seen that

$$
\begin{aligned}
& \operatorname{det} \mathcal{D}_{112}\left(h^{*}\right)=\operatorname{det} \mathcal{D}_{211}\left(h^{*}\right) \neq 0, \forall h^{*} \leq 2.3 ; \\
& \operatorname{det} \mathcal{D}_{122}\left(h^{*}\right)=\operatorname{det} \mathcal{D}_{221}\left(h^{*}\right) \neq 0, \forall h^{*} \leq 2.3
\end{aligned}
$$

Remark 3.4. If we only pursue the stability of closed-loop system without chasing better performance, then we can merely employ the one-step approach to estimate the system parameters and update the control law synchronously. In this case, conditions (3.2)-(3.3) can be simplified as

$$
h \leq \min \left\{1, h_{0}\right\}, \quad \frac{c_{2} \gamma_{0}}{1-\gamma_{0}}+8 c_{2}\left(2 \lambda+\lambda_{1} h\right) h \frac{\left(1+\gamma_{0}\right)^{2}}{\left(1-\gamma_{0}\right)^{2}}<\frac{1}{2} .
$$

When calculating the LQ performance index and analyzing its upper bound, in order to simplify the calculation and get a smaller upper bound, we introduce condition (3.2) and (3.3) for selecting estimation step and control step.

As for condition (3.2), the first inequality is deduced to simplify the proof of Lemma 4.4 given below, which is a sufficient condition. Hence, in some circumstances, $h^{*}$ may be larger than that given by (3.2). By the first inequality of (3.2) and the definition of $\gamma_{0}$, we have $2 c_{1} h^{*} \mathrm{e}^{c_{1} h^{*}}<2 c_{1} \mathrm{e}^{c_{1}} h^{*}<\sqrt{2}-1$. As a result,

$$
\frac{c_{2} \gamma_{0}}{1-\gamma_{0}} \leq(2+\sqrt{2}) c_{1} c_{2} \mathrm{e}^{c_{1}} h^{*}
$$

According to the solution of quadratic inequality

$$
5 c_{2} h^{*}\left(2 \lambda+\lambda_{1} h^{*}\right)+(2+\sqrt{2}) c_{1} c_{2} \mathrm{e}^{c_{1}} h^{*}<\frac{1}{2}
$$

we know that for any

$$
h^{*} \in\left(0, \frac{1}{2\left((2+\sqrt{2}) c_{1} c_{2} \mathrm{e}^{c_{1}}+10 \lambda c_{2}\right)+\sqrt{10 \lambda_{1} c_{2}}}\right),
$$

the second inequality in (3.2) holds.

As for condition (3.3), by the DS-approach we require $h \leq h^{*}$. Meanwhile, we need $h \leq \frac{9-4 \sqrt{5}}{2 c_{1} \mathrm{e}^{c_{1}}}$ so as to simplify the calculation (see the proof of Thm. 3.1 in Sect. 4.2). In regard to the second inequality in (3.3), by condition $(3.2), h^{*} \leq \frac{\sqrt{2}-1}{2 c_{1} \mathrm{e}^{c_{1}}}$, which together with inequality $\mathrm{e}^{x}<1+2 x, \forall x \in(0,1]$, gives

$$
\mathrm{e}^{c_{1} h}<1+2 c_{1} h, \quad \mathrm{e}^{2 c_{1} h}<1+4 c_{1} h
$$

Hence, the range of control step $h$ can be solved through the following inequality

$$
2 c_{1} h^{2}+h \leq \frac{\min _{i \neq k}\left\|\mathcal{D}_{i j k}\left(h^{*}\right)\right\|}{32 c_{1}^{3} h^{* 2} \mathrm{e}^{2 c_{1} h^{*}}}
$$

which is a key point in the proof of Lemma 4.3 of the next section. 
Remark 3.5. According to Lemma 4.2 given below, parameters $\lambda$ and $\lambda_{1}$ represent the transition probability of system parameter $\theta_{t}$. Precisely, they denote the probability upper bound of $\theta_{t}$ jumping from parameter $i$ to another parameter $j$ on an interval with length $h^{*}$. By (3.2), when the transition probability of $\theta_{t}$ is sufficiently small (that is, $\lambda$ and $\lambda_{1}$ are sufficiently small), the range of $h^{*}$ may be greater than that given by (3.2).

\section{Proof of Theorem 3.1}

\subsection{Basic lemmas}

In order to prove Theorem 3.1 we need the following lemmas. Lemma 4.1 comes from [24] and is listed here just for self-containedness. The proofs of Lemma 4.2-4.4 are put into Appendix A.

Lemma 4.1 ([24]). Suppose $\theta_{t}$ is a stationary Markov process taking values in a finite state $\mathcal{S}=\{1, \ldots, N\}$ with transition probability given by (1.2) and (1.3). If functions $f\left(\theta_{t}\right): \mathbb{R} \rightarrow \mathbb{R}$ and $g\left(\theta_{t}\right): \mathbb{R} \rightarrow \mathbb{R}^{n \times n}$ are both measurable with respect to $\sigma\left\{\theta_{s}, s \leq t\right\}$, then when $s-s_{0} \leq 1$, we have

$$
E\left[\left\|g\left(\theta_{s}\right)-g\left(\theta_{s_{0}}\right)\right\| \int_{s_{0}}^{s} f\left(\theta_{\mu}\right) \mathrm{d} \mu \mid \theta_{s_{0}}=i_{0}\right] \leq \max _{j \neq i_{0}}\left\|g(j)-g\left(i_{0}\right)\right\| \max _{l \in \mathcal{S}} f(l) \lambda\left(s-s_{0}\right)^{2},
$$

where $\lambda$ is given by (3.5).

Remark 4.1. Lemma 4.1 can be explained roughly in this way. In the case where $\theta_{t}$ has no jump on interval $\left[s_{0}, s\right]$, the left hand side of (4.1) is obviously equal to 0 . In the case where $\theta_{t}$ jumps with small probability on interval $\left[s_{0}, s\right]$, the left hand side of (4.1) is also small.

Lemma 4.2. Suppose $\left\{\theta_{t}, \mathcal{F}_{t}^{\theta}\right\}$ is a Markov process defined on probability space $(\Omega, \mathcal{F}, P)$ taking values in a finite set $\mathcal{S}=\{1, \ldots, N\}$ with transition probability given by (1.2) and (1.3), where $\mathcal{F}_{t}^{\theta}=\sigma\left(\theta_{s}, s \leq t\right)$. If function $f\left(\theta_{t}\right): \mathbb{R} \rightarrow \mathbb{R}$ and $g\left(\theta_{t}\right): \mathbb{R} \rightarrow \mathbb{R}^{n \times n}$ are measurable with respect to $\mathcal{F}_{t}^{\theta}$, then when $h \leq 1$, we have

$E\left[\left\|g\left(\theta_{s^{\prime}}\right)-g\left(\hat{\theta}_{s_{0}}\right)\right\| \int_{s^{\prime}}^{s} f\left(\theta_{\mu}\right) \mathrm{d} \mu I_{\mathcal{G}_{s_{0}-h}} \mid \theta_{s_{0}-h}=i_{0}\right] \leq\left(\lambda+\lambda_{1} h\right) h\left(s-s^{\prime}\right) \max _{i, j \in \mathcal{S}}\|g(i)-g(j)\| \max _{k \in \mathcal{S}} f(k), \quad \forall s^{\prime} \in\left(s_{0}, s\right)$,

where $i_{0}, k \in \mathcal{S} ; \lambda$ and $\lambda_{1}$ are described by (3.5), and

$$
\mathcal{G}_{s_{0}-h}=\left\{\omega: \omega \in \Omega, \theta_{t}(\omega) \text { jumps in }\left(s_{0}-h, s_{0}\right)\right\} .
$$

Remark 4.2. Lemmas 4.1 and 4.2 both display the expectation estimate of stochastic functions with respect to Markov process, but Lemma 4.2 is more precise. In Lemma 4.1, when $\theta_{s_{0}}=i_{0}, \theta_{s}=i_{0}$, because of term $\left\|g\left(\theta_{s}\right)-g\left(\theta_{s_{0}}\right)\right\|$, the left hand is obviously equal to 0 . However, Lemma 4.2 describes a different case, which needs us to estimate the expectation on $\mathcal{G}_{s_{0}-h}$, namely, $\theta_{t}$ jumps at least once in the time interval $\left[s_{0}-h, s_{0}\right]$. Intuitively, this probability is small.

Lemma 4.3. For the system (1.1)-(1.2) with Assumptions 3.1 and 3.2, if the estimation step $h^{*}$ and the control step $h$ satisfy (3.1)-(3.3), then $\gamma_{2}$ defined by (3.9) is positive.

Remark 4.3. By condition $(3.3), h \leq \frac{9-4 \sqrt{5}}{2 c_{1} \mathrm{e}^{c_{1}}}, \frac{1}{1-d_{1}} \leq 1.06, \ln \frac{1}{1-d_{1}}<0.06$. Thus, the second inequality in condition (3.3) ensures that

$$
\mathrm{e}^{c_{1} h}+2 c_{1} h \mathrm{e}^{2 c_{1} h}\left(\frac{1}{2}+\ln \frac{1}{1-d_{1}}\right)<1+\frac{\left\|\mathcal{D}_{k i j}\left(h^{*}\right)\right\|}{8 c_{1}^{2} h^{* 2} \mathrm{e}^{2 c_{1} h^{*}}}, \quad \forall i \neq k .
$$

So, by (A.10) one can get $\left\|2 \mathcal{D}_{k i j}^{-1}\left(h^{*}\right) \int_{0}^{1} \mathrm{e}^{(1-t)\left(A_{i}-B_{i} L_{j}\right) h^{*}} \Delta_{i j} F_{i j}^{t N^{*}} \mathrm{~d} t\right\|<1$. 
Lemma 4.4. Under the conditions of Theorem 3.1, for any given integers $k=0,1, \ldots, l=0,1, \ldots, N^{*}-1$, and any real number $t \in\left[k h^{*}+l h, k h^{*}+l h+h\right)$, when $\theta_{t}$ has no jump on the time interval $\left[(k-1) h^{*}, k h^{*}\right)$, we have

$$
\sum_{k=0}^{K-1} \sum_{l=0}^{N^{*}-1} E \int_{k h^{*}+l h}^{k h^{*}+(l+1) h}\left\|x_{\left(k h^{*}+l h\right)}\right\|^{2} \mathrm{~d} s \leq \frac{13}{2} \gamma_{1}\left(\frac{2}{\gamma_{2}}+1\right)^{2} K h^{* 2}+\frac{9}{2} K h^{* 2} \max _{i \in \mathcal{S}} \operatorname{tr}\left(C_{i}^{T} C_{i}\right)
$$

where $\gamma_{1}$ and $\gamma_{2}$ are defined by (3.8) and (3.9), respectively.

\subsection{Proof of Theorem 3.1}

The proof of Theorem 3.1 is divided into three parts.

\section{Part 1: Closed-loop system and its increment property}

Firstly we give the closed-loop system. Let

$$
k_{t}=\left\lceil\frac{t}{h^{*}}\right\rceil, \quad l_{t}=\left\lceil\frac{t-k_{t} h^{*}}{h}\right\rceil, \quad t^{\prime}=k_{t} h^{*}+l_{t} h
$$

where $\lceil x\rceil$ denotes the maximum integer less than $x$.

From the notations of (4.2), one can easily see that $t \in\left[t^{\prime}, t^{\prime}+h\right)$. And by the remarks under (2.4), we have $h^{*}=N^{*} h$ for some positive integer $N^{*}$. Thus, $l_{t} \in\left\{0,1, \ldots, N^{*}-1\right\}$ is bounded.

Applying the SD-based adaptive control (2.4) to the system (1.1), we have the following closed-loop system:

$$
\begin{aligned}
\mathrm{d} x_{t}= & A\left(\theta_{t}\right) x_{t} \mathrm{~d} t-B\left(\theta_{t}\right) L\left(\hat{\theta}_{k_{t} h^{*}}\right) x_{t^{\prime}} \mathrm{d} t+C\left(\theta_{t}\right) \mathrm{d} W_{t} \\
= & {\left[A\left(\theta_{t}\right)-B\left(\theta_{t}\right) L\left(\hat{\theta}_{k_{t} h^{*}}\right)\right] x_{t^{\prime}} \mathrm{d} t+A\left(\theta_{t}\right)\left(x_{t}-x_{t^{\prime}}\right) \mathrm{d} t+C\left(\theta_{t}\right) \mathrm{d} W_{t} } \\
= & A_{1}\left(\theta_{t}\right) x_{t} \mathrm{~d} t+B\left(\theta_{t}\right)\left(L\left(\theta_{t}\right)-L\left(\hat{\theta}_{k_{t} h^{*}}\right)\right) x_{t} \mathrm{~d} t \\
& +B\left(\theta_{t}\right) L\left(\hat{\theta}_{k_{t} h^{*}}\right)\left(x_{t}-x_{t^{\prime}}\right) \mathrm{d} t+C\left(\theta_{t}\right) \mathrm{d} W_{t}, \quad t \in\left[t^{\prime}, t^{\prime}+h\right),
\end{aligned}
$$

where $A_{1}\left(\theta_{t}\right)=A\left(\theta_{t}\right)-B\left(\theta_{t}\right) L\left(\theta_{t}\right)$. By (4.3),

$$
x_{t}-x_{t^{\prime}}=\int_{t^{\prime}}^{t} A\left(\theta_{s}\right)\left(x_{s}-x_{t^{\prime}}\right) \mathrm{d} s+\int_{t^{\prime}}^{t}\left[A\left(\theta_{s}\right)-B\left(\theta_{s}\right) L\left(\hat{\theta}_{k_{t} h^{*}}\right)\right] \mathrm{d} s x_{t^{\prime}}+\int_{t^{\prime}}^{t} C\left(\theta_{s}\right) \mathrm{d} W_{s} .
$$

Hence,

$$
\left\|x_{t}-x_{t^{\prime}}\right\| \leq 2 c_{1} h\left\|x_{t^{\prime}}\right\|+c_{1} \int_{t^{\prime}}^{t}\left\|x_{s}-x_{t^{\prime}}\right\| \mathrm{d} s+\left\|\int_{t^{\prime}}^{t} C\left(\theta_{s}\right) \mathrm{d} W s\right\|, \quad \forall t \in\left[t^{\prime}, t^{\prime}+h\right],
$$

where $c_{1}$ is described by (3.6). Then, by Gronwall lemma [5] we get

$$
\begin{gathered}
\left\|x_{t}-x_{t^{\prime}}\right\| \leq d_{1}\left\|x_{t^{\prime}}\right\|+d_{2}(t), \quad \forall t \in\left[t^{\prime}, t^{\prime}+h\right] \\
\left\|x_{t}\right\| \leq\left(1+d_{1}\right)\left\|x_{t^{\prime}}\right\|+d_{2}(t), \quad\left\|x_{t^{\prime}}\right\| \leq \frac{1}{1-d_{1}}\left\|x_{t}\right\|+\frac{d_{2}(t)}{1-d_{1}}
\end{gathered}
$$

where

$$
\begin{aligned}
d_{1} & =2 c_{1} h \mathrm{e}^{c_{1} h} \\
d_{2}(t) & =c_{1} \mathrm{e}^{c_{1} h} \int_{t^{\prime}}^{t}\left\|\int_{t^{\prime}}^{s} C\left(\theta_{\mu}\right) \mathrm{d} W_{\mu}\right\| \mathrm{d} s+\left\|\int_{t^{\prime}}^{t} C\left(\theta_{s}\right) \mathrm{d} W_{s}\right\| .
\end{aligned}
$$


Let

$$
\gamma_{4}=2 c_{4}+c_{4} c_{1}^{2} \mathrm{e}^{2 c_{1} h} h^{2}, \quad c_{4}=\max _{i \in \mathcal{S}} \operatorname{tr}\left(C_{i}^{T} C_{i}\right) .
$$

Then, by Schwarz inequality and Fubini theorem [17] we have

$$
\begin{aligned}
E d_{2}^{2}(t) & \leq 2 h c_{1}^{2} \mathrm{e}^{2 c_{1} h} \int_{t^{\prime}}^{t} E\left\|\int_{t^{\prime}}^{s} C\left(\theta_{\mu}\right) \mathrm{d} W_{\mu}\right\|^{2} \mathrm{~d} s+2 E\left\|\int_{t^{\prime}}^{t} C\left(\theta_{s}\right) \mathrm{d} W_{s}\right\|^{2} \\
& =2 h c_{1}^{2} \mathrm{e}^{2 c_{1} h} \int_{t^{\prime}}^{t} \int_{t^{\prime}}^{s} \operatorname{tr}\left(C^{T}\left(\theta_{\mu}\right) C\left(\theta_{\mu}\right)\right) \mathrm{d} \mu \mathrm{d} s+2 \int_{t^{\prime}}^{t} \operatorname{tr}\left(C^{T}\left(\theta_{s}\right) C\left(\theta_{s}\right)\right) \mathrm{d} s \\
& \leq \gamma_{4} h .
\end{aligned}
$$

\section{Part 2: Stability of the closed-loop system}

According to [12] and Assumption 3.1, the coupled Riccati equations

$$
A_{1, i}^{T} K_{i}+K_{i} A_{1, i}+\sum_{j} \lambda_{i, j} K_{j}=-I
$$

have a unique symmetric positive definite solution set $\left\{K_{i}, i \in \mathcal{S}\right\}$. Construct $K\left(\theta_{t}\right)$ such that when $\theta_{t}=i$, $K\left(\theta_{t}\right)=K_{i}$. Similar to equation (2.29) of [25], by (4.4) and the SD-based adaptive control (2.4) we get

$$
\begin{aligned}
\widetilde{\mathcal{A}}\left(x_{t}^{T} K\left(\theta_{t}\right) x_{t}\right) \triangleq & \lim _{\Delta \rightarrow 0} \frac{1}{\Delta}\left(E\left[x_{t+\Delta}^{T} K\left(\theta_{t+\Delta}\right) x_{t+\Delta} \mid \theta_{t}\right]-x_{t}^{T} K\left(\theta_{t}\right) x_{t}\right) \\
= & x_{t}^{T}\left(A_{1}^{T}\left(\theta_{t}\right) K\left(\theta_{t}\right)+K\left(\theta_{t}\right) A_{1}\left(\theta_{t}\right)+\sum_{j} \lambda_{\theta_{t}, j} K_{j}\right) x_{t}+\operatorname{tr}\left(C^{T}\left(\theta_{t}\right) K\left(\theta_{t}\right) C\left(\theta_{t}\right)\right) \\
& +2 x_{t}^{T}\left(L\left(\theta_{t}\right)-L\left(\hat{\theta}_{k_{t} h^{*}}\right)\right)^{T} B^{T}\left(\theta_{t}\right) K\left(\theta_{t}\right) x_{t}+2\left(x_{t}-x_{t^{\prime}}\right)^{T} L^{T}\left(\hat{\theta}_{k_{t} h^{*}}\right) B^{T}\left(\theta_{t}\right) K\left(\theta_{t}\right) x_{t} \\
= & -\left\|x_{t}\right\|^{2}+\operatorname{tr}\left(C^{T}\left(\theta_{t}\right) K\left(\theta_{t}\right) C\left(\theta_{t}\right)\right)+2 x_{t}^{T}\left(L\left(\theta_{t}\right)-L\left(\theta_{t^{\prime}}\right)\right)^{T} B^{T}\left(\theta_{t}\right) K\left(\theta_{t}\right) x_{t} \\
& +2 x_{t}^{T}\left(L\left(\theta_{t^{\prime}}\right)-L\left(\hat{\theta}_{k_{t} h^{*}}\right)\right)^{T} B^{T}\left(\theta_{t}\right) K\left(\theta_{t}\right) x_{t}+2\left(x_{t}-x_{t^{\prime}}\right)^{T} L^{T}\left(\hat{\theta}_{k_{t} h^{*}}\right) B^{T}\left(\theta_{t}\right) K\left(\theta_{t}\right) x_{t},
\end{aligned}
$$

where $\widetilde{\mathcal{A}}$ is an infinitesimal operator of associated process $\left\{\theta_{t}, x_{t}\right\}$.

We now analyze the last three terms on the right hand of the above equation (4.12).

For the last term on the right hand of (4.12), by (4.5)-(4.6) we have

$$
\begin{aligned}
2\left(x_{t}-x_{t^{\prime}}\right)^{T} L^{T}\left(\hat{\theta}_{k_{t} h^{*}}\right) B^{T}\left(\theta_{t}\right) K\left(\theta_{t}\right) x_{t} & \leq 2 c_{2}\left\|x_{t}-x_{t^{\prime}}\right\|\left\|x_{t}\right\| \\
& \leq 2 c_{2}\left(\frac{d_{1}}{1-d_{1}}\left\|x_{t}\right\|+\frac{d_{2}(t)}{1-d_{1}}\right)\left\|x_{t}\right\|=2 \alpha\left\|x_{t}\right\|^{2}+2 \beta(t)\left\|x_{t}\right\|,
\end{aligned}
$$

where $c_{2}, d_{1}, d_{2}(t)$ are determined by (3.6), (4.7), (4.8), respectively, and

$$
\alpha=\frac{c_{2} d_{1}}{1-d_{1}}, \quad \beta(t)=\frac{c_{2} d_{2}(t)}{1-d_{1}} .
$$


To study the third term on the right hand of (4.12), let

$$
\Phi(s)=\left(L\left(\theta_{s}\right)-L\left(\theta_{s^{\prime}}\right)\right)^{T} B^{T}\left(\theta_{s}\right) K\left(\theta_{s}\right)
$$

Then, for any positive integer $K$, by (4.6), (4.10), Fubini theorem [17] and Lemma 4.1, we have

$$
\begin{aligned}
E \int_{0}^{K h} x_{s}^{T} \Phi(s) x_{s} \mathrm{~d} s \leq & E \int_{0}^{K h}\|\Phi(s)\|\left(2\left(1+d_{1}\right)^{2}\left\|x_{s^{\prime}}\right\|^{2}+2 d_{2}^{2}(s)\right) \mathrm{d} s \\
\leq & \sum_{k=0}^{K-1} \sum_{i=1}^{N} \int_{k h}^{(k+1) h} E\left[\|\Phi(s)\| 2\left(1+d_{1}\right)^{2}\left\|x_{k h}\right\|^{2} \mathrm{~d} s|| \theta_{k h}=i\right] P\left(\theta_{k h}=i\right) \\
& +2 \sum_{k=0}^{K-1} \sum_{i=1}^{N} \int_{k h}^{(k+1) h} E\left[\|\Phi(s)\| d_{2}^{2}(s) \mathrm{d} s \mid \theta_{k h}=i\right] P\left(\theta_{k h}=i\right) \\
\leq & 2 c_{2}\left(1+d_{1}\right)^{2} \lambda h \sum_{k=0}^{K-1} \int_{k h}^{(k+1) h} E\left\|x_{k h}\right\|^{2} \mathrm{~d} s+2 \lambda \gamma_{4} c_{2} K h^{3} \\
\leq & \frac{4\left(1+d_{1}\right)^{2}}{\left(1-d_{1}\right)^{2}} c_{2} \lambda h \sum_{k=0}^{K-1} \int_{k h}^{(k+1) h}\left(E\left\|x_{s}\right\|^{2}+E d_{2}^{2}(s)\right) \mathrm{d} s+2 \lambda \gamma_{4} c_{2} K h^{3} \\
\leq & \frac{4\left(1+d_{1}\right)^{2}}{\left(1-d_{1}\right)^{2}} c_{2} \lambda h E \int_{0}^{K h}\left\|x_{s}\right\|^{2} \mathrm{~d} s+\left(\frac{4\left(1+d_{1}\right)^{2}}{\left(1-d_{1}\right)^{2}}+2\right) \lambda \gamma_{4} c_{2} K h^{3} .
\end{aligned}
$$

Similarly, it can be shown that (4.14) still holds with $K h$ replaced by any given $t>0$. Noticing that

$$
\frac{\left(1+d_{1}\right)^{2}}{\left(1-d_{1}\right)^{2}} \leq \frac{5}{4}, \forall h \leq \frac{9-4 \sqrt{5}}{2 c_{1} \mathrm{e}^{c_{1}}}
$$

by (4.14) we have the following estimates for the third term on the right hand of (4.12):

$$
E \int_{0}^{t} x_{s}^{T}\left(L\left(\theta_{s}\right)-L\left(\theta_{s^{\prime}}\right)\right)^{T} B^{T}\left(\theta_{s}\right) K\left(\theta_{s}\right) x_{s} \mathrm{~d} s \leq 5 \lambda h c_{2} E \int_{0}^{t}\left\|x_{s}\right\|^{2} \mathrm{~d} s+7 \lambda \gamma_{4} c_{2} h^{2} t
$$

To study the forth term on the right hand of (4.12), let

$$
\begin{aligned}
& \Psi(t, k)=\left(L\left(\theta_{t^{\prime}}\right)-L\left(\hat{\theta}_{k h^{*}}\right)\right)^{T} B^{T}\left(\theta_{t}\right) K\left(\theta_{t}\right), \\
& \mathcal{G}_{(k-1) h^{*}}=\left\{\omega: \omega \in \Omega, \theta_{t}(\omega) \text { jumps on interval }\left[(k-1) h^{*}, k h^{*}\right)\right\}, \\
& \overline{\mathcal{G}}_{(k-1) h^{*}}=\left\{\omega: \omega \in \Omega, \theta_{t}(\omega) \text { does not jump on interval }\left[(k-1) h^{*}, k h^{*}\right)\right\} .
\end{aligned}
$$


When system (1.1)-(1.2) jumps on interval $\left[(k-1) h^{*}, k h^{*}\right)$, by Lemma 4.2 we have the following estimates for the forth term on the right hand of (4.12):

$$
\begin{aligned}
E \int_{0}^{K h^{*}} x_{s}^{T} \Psi\left(s, k_{s}\right) x_{s} I_{\mathcal{G}_{\left(k_{s}-1\right) h^{*}} \mathrm{~d} s \leq} & \sum_{k=0}^{K-1} \sum_{l=0}^{N^{*}-1} E \int_{k h^{*}+l h}^{k h^{*}+(l+1) h}\|\Psi(s, k)\|\left(2\left(1+d_{1}\right)^{2}\left\|x_{s^{\prime}}\right\|^{2}+2 d_{2}^{2}(s)\right) I_{\mathcal{G}_{(k-1) h^{*}}} \mathrm{~d} s \\
\leq & \sum_{k=0}^{K-1} \sum_{l=0}^{N^{*}-1} \sum_{i=1}^{N} \int_{k h^{*}+l h}^{k h^{*}+(l+1) h} E\left[\|\Psi(s, k)\| I_{\left.\mathcal{G}_{(k-1) h^{*}} \mid \theta_{(k-1) h^{*}}=i\right]}\right. \\
& \times 2\left(1+d_{1}\right)^{2}\left\|x_{s^{\prime}}\right\|^{2} \mathrm{~d} s P\left(\theta_{(k-1) h^{*}}=i\right) \\
& +\sum_{k=0}^{K-1} \sum_{l=0}^{N^{*}-1} E \int_{k h^{*}+l h}^{k h^{*}+(l+1) h}\|\Psi(s, k)\| I_{\mathcal{G}_{(k-1) h^{*}} 2 d_{2}^{2}(s) \mathrm{d} s} \\
\leq & \frac{4\left(1+d_{1}\right)^{2}}{\left(1-d_{1}\right)^{2}} c_{2}\left(\lambda h^{*}+\lambda_{1} h^{* 2}\right) \sum_{k=0}^{K-1} \sum_{l=0}^{N^{*}-1} E \int_{k h^{*}+l h}^{k h^{*}+(l+1) h}\left(\left\|x_{s}\right\|^{2}+d_{2}^{2}(s)\right) \mathrm{d} s \\
& +2 \gamma_{4} c_{2}\left(\lambda+\lambda_{1} h^{*}\right) h h^{* 2} \\
\leq & 5 c_{2}\left(\lambda+\lambda_{1} h^{*}\right) h^{*} E \int_{0}^{K h^{*}}\left\|x_{s}\right\|^{2} \mathrm{~d} s+7 h \gamma_{4} c_{2}\left(\lambda+\lambda_{1} h^{*}\right) K h^{* 2}
\end{aligned}
$$

and

$$
E \int_{0}^{t} x_{s}^{T} \Psi\left(s, k_{s}\right) x_{s} I_{\mathcal{G}_{\left(k_{s}-1\right) h^{*}}} \mathrm{~d} s \leq 5 c_{2}\left(\lambda+\lambda_{1} h^{*}\right) h^{*} E \int_{0}^{t}\left\|x_{s}\right\|^{2} \mathrm{~d} s+7 h \gamma_{4} c_{2}\left(\lambda+\lambda_{1} h^{*}\right) h^{*} t .
$$

When the system $(1.1)-(1.2)$ has no jump on interval $\left[(k-1) h^{*}, k h^{*}\right)$, by Remark $4.3,\left(1+d_{1}\right)^{2} \leq \frac{5}{4}$, and Lemma 4.4, we have the following estimates for the forth term on the right hand of (4.12):

$$
\begin{aligned}
& E \int_{0}^{K h^{*}} x_{s}^{T} \Psi\left(s, k_{s}\right) x_{s} I_{\overline{\mathcal{G}}_{\left(k_{s}-1\right) h^{*}} \mathrm{~d} s \leq} \sum_{k=0}^{K-1} \sum_{l=0}^{N^{*}-1} E \int_{k h^{*}+l h}^{k h^{*}+(l+1) h}\|\Psi(s, k)\|\left\|x_{s}\right\|^{2} I_{\left[\left\|x_{s}\right\|^{2} \leq 2\left(1+d_{1}\right)^{2}\left\|x_{\left(k h^{*}+l h\right)}\right\|^{2}+2 d_{2}^{2}(s)\right]} \mathrm{d} s \\
& \leq 2 c_{2}\left(1+d_{1}\right)^{2} \sum_{k=0}^{K-1} \sum_{l=0}^{N^{*}-1} E \int_{k h^{*}+l h}^{k h^{*}+(l+1) h}\left\|x_{\left(k h^{*}+l h\right)}\right\|^{2} \mathrm{~d} s \\
&+2 c_{2} \sum_{k=0}^{K-1} \sum_{l=0}^{N^{*}-1} E \int_{k h^{*}+l h}^{k h^{*}+(l+1) h} d_{2}^{2}(s) \mathrm{d} s \\
& \leq 17 c_{2} \gamma_{1} \gamma_{3}^{2} K h^{* 2}+12 \gamma_{4} c_{2} K h^{* 2}+2 \gamma_{4} c_{2} h K h^{*}
\end{aligned}
$$

and

$$
E \int_{0}^{t} x_{s}^{T} \Psi\left(s, k_{s}\right) x_{s} I_{\overline{\mathcal{G}}_{\left(k_{s}-1\right) h^{*}}} \mathrm{~d} s \leq 17 c_{2} \gamma_{1} \gamma_{3}^{2} h^{*} t+12 \gamma_{4} c_{2} h^{*} t+2 \gamma_{4} c_{2} h t
$$

where $\gamma_{1}$ and $\gamma_{2}$ are given by (3.8) and (3.9), respectively, and

$$
\gamma_{3}=\frac{2}{\gamma_{2}}+1
$$


According to (4.12) and the Dynkin formula [16], by (4.13), (4.15), (4.17), (4.19) and the Jensen inequality [4], we have

$$
\begin{aligned}
E\left(x_{t}^{T} K\left(\theta_{t}\right) x_{t}\right)-E\left(x_{0}^{T} K\left(\theta_{0}\right) x_{0}\right) \leq & -E \int_{0}^{t}\left\|x_{s}\right\|^{2} \mathrm{~d} s+E \int_{0}^{t} \operatorname{tr}\left(C^{T}\left(\theta_{s}\right) K\left(\theta_{s}\right) C\left(\theta_{s}\right)\right) \mathrm{d} s \\
& +\left(20 \lambda+10 \lambda_{1} h^{*}\right) c_{2} h^{*} E \int_{0}^{t}\left\|x_{s}\right\|^{2} \mathrm{~d} s \\
& +\left(28 \lambda+14 \lambda_{1} h^{*}\right) c_{2} h^{*} \gamma_{4} h t+34 \gamma_{1} c_{2} \gamma_{3}^{2} h^{*} t \\
& +24 c_{2} \gamma_{4} h^{*} t+4 \gamma_{4} c_{2} h t+2 E \int_{0}^{t} \alpha\left\|x_{s}\right\|^{2} \mathrm{~d} s+2 E \int_{0}^{t} \beta(s)\left\|x_{s}\right\| \mathrm{d} s \\
\leq & -\left(1-\left(20 \lambda+10 \lambda_{1} h^{*}\right) c_{2} h^{*}-2 \alpha\right) E \int_{0}^{t}\left\|x_{s}\right\|^{2} \mathrm{~d} s \\
& +E \int_{0}^{t} \operatorname{tr}\left(C^{T}\left(\theta_{s}\right) K\left(\theta_{s}\right) C\left(\theta_{s}\right)\right) \mathrm{d} s \\
& +\left(28 \lambda+14 \lambda_{1} h^{*}\right) c_{2} h^{*} \gamma_{4} h t+34 \gamma_{1} c_{2} \gamma_{3}^{2} h^{*} t+24 c_{2} \gamma_{4} h^{*} t+4 \gamma_{4} c_{2} h t \\
& +2\left(E \int_{0}^{t} \beta^{2}(s) \mathrm{d} s\right)^{\frac{1}{2}}\left(E \int_{0}^{t}\left\|x_{s}\right\|^{2} \mathrm{~d} s\right)^{\frac{1}{2}} .
\end{aligned}
$$

Since $\left\|W_{t}-W_{t^{\prime}}\right\|$ are mutually independent, by (4.10), it is obvious that

$$
\limsup _{t \rightarrow \infty} \frac{1}{t} E \int_{0}^{t} d_{2}^{2}(s) \mathrm{d} s=O(h), \quad \limsup _{t \rightarrow \infty} \frac{1}{t} E \int_{0}^{t} \beta^{2}(s) \mathrm{d} s=O(h) .
$$

Therefore,

$$
\begin{aligned}
\limsup _{t \rightarrow \infty}\left(\frac{1}{t} E\left(x_{t}^{T} K\left(\theta_{t}\right) x_{t}\right)\right. & \left.+\left(1-\left(20 \lambda+10 \lambda_{1} h^{*}\right) c_{2} h^{*}-2 \alpha\right) \frac{1}{t} E \int_{0}^{t}\left\|x_{s}\right\|^{2} \mathrm{~d} s\right) \\
\leq \limsup _{t \rightarrow \infty} \frac{1}{t} E \int_{0}^{t} \operatorname{tr}\left(C^{T}\left(\theta_{s}\right) K\left(\theta_{s}\right) C\left(\theta_{s}\right)\right) \mathrm{d} s+34 \gamma_{1} c_{2} \gamma_{3}^{2} h^{*} & \\
& +24 c_{2} c_{4} h^{*}+O(h)+O\left(h^{\frac{1}{2}}\right)\left(\limsup _{t \rightarrow \infty} \frac{1}{t} E \int_{0}^{t}\left\|x_{s}\right\|^{2} \mathrm{~d} s\right)^{\frac{1}{2}} .
\end{aligned}
$$

Noticing

$$
\alpha=\frac{2 c_{1} c_{2} h \mathrm{e}^{c_{1} h}}{1-2 c_{1} h \mathrm{e}^{c_{1} h}} \leq \frac{c_{2} \gamma_{0}}{1-\gamma_{0}}
$$

by (3.2) we have

$$
1-\left(20 \lambda+10 \lambda_{1} h^{*}\right) c_{2} h^{*}-2 \alpha \geq 1-\left(20 \lambda+10 \lambda_{1} h^{*}\right) c_{2} h^{*}-\frac{2 c_{2} \gamma_{0}}{1-\gamma_{0}}>0
$$


This together with (4.22) gives

$$
\begin{aligned}
\limsup _{t \rightarrow \infty} \frac{1}{t} E \int_{0}^{t}\left\|x_{s}\right\|^{2} \mathrm{~d} s \leq & \frac{2}{\gamma_{5}} \limsup _{t \rightarrow \infty} \frac{1}{t} E \int_{0}^{t} \operatorname{tr}\left(C^{T}\left(\theta_{s}\right) K\left(\theta_{s}\right) C\left(\theta_{s}\right)\right) \mathrm{d} s+\frac{48}{\gamma_{5}} c_{2} c_{4} h^{*} \\
& +\frac{68}{\gamma_{5}} \gamma_{1} c_{2} \gamma_{3}^{2} h^{*}+O(h)<\infty
\end{aligned}
$$

where $\gamma_{5}=1-32 c_{2} h^{*}\left(2 \lambda+\lambda_{1} h^{*}\right)-\frac{2 c_{2} \gamma_{0}}{1-\gamma_{0}}$.

Thus, the closed-loop system is stable.

\section{Part 3: On the LQ performance index}

Denote

$$
u_{t}^{*}=-L\left(\theta_{t}\right) x_{t}
$$

Then, similar to (4.13), (4.15), (4.17), (4.19) and (4.23) we have

$$
\begin{aligned}
\limsup _{t \rightarrow \infty} \frac{1}{t} E \int_{0}^{t}\left(u_{s}-u_{s}^{*}\right)^{T} R\left(\theta_{s}\right)\left(u_{s}-u_{s}^{*}\right) \mathrm{d} s= & \limsup _{t \rightarrow \infty} \frac{1}{t} E \int_{0}^{t}\left[x_{s}^{T}\left(L\left(\theta_{s}\right)-L\left(\hat{\theta}_{s^{*}}\right)\right)^{T} R\left(\theta_{s}\right)\left(L\left(\theta_{s}\right)-L\left(\hat{\theta}_{s^{*}}\right)\right) x_{s}\right. \\
& +2 x_{s}^{T}\left(L\left(\theta_{s}\right)-L\left(\hat{\theta}_{s^{*}}\right)\right)^{T} R\left(\theta_{s}\right) L\left(\hat{\theta}_{s^{*}}\right)\left(x_{s}-x_{s^{\prime}}\right) \\
& \left.+\left(x_{s}-x_{s^{\prime}}\right)^{T} L^{T}\left(\hat{\theta}_{s^{*}}\right) R\left(\theta_{s}\right) L\left(\hat{\theta}_{s^{*}}\right)\left(x_{s}-x_{s^{\prime}}\right)\right] \mathrm{d} s \\
\leq & \left(15 c_{3} \lambda h+5 c_{3}\left(\lambda+\lambda_{1} h^{*}\right) h^{*}\right) \limsup _{t \rightarrow \infty} \frac{1}{t} E \int_{0}^{t}\left\|x_{s}\right\|^{2} \mathrm{~d} s+17 \gamma_{1} \gamma_{3}^{2} c_{3} h^{*} \\
& \left.\frac{2 c_{5} d_{1}}{1-d_{1}} \limsup _{t \rightarrow \infty} \frac{1}{t} E \int_{0}^{t}\left\|x_{s}\right\|^{2} \mathrm{~d} s+O\left(h^{\frac{1}{2}}\right)\left(\limsup _{t \rightarrow \infty} \frac{1}{t} E \int_{0}^{t}\left\|x_{s}\right\|^{2} \mathrm{~d} s\right)\right)^{\frac{1}{2}} \\
& +\frac{2 c_{6} d_{1}^{2}}{\left(1-d_{1}\right)^{2}} \limsup _{t \rightarrow \infty} \frac{1}{t} E \int_{0}^{t}\left\|x_{s}\right\|^{2} \mathrm{~d} s+O\left(h^{*}\right)+O(h) \\
\leq & \frac{340}{\gamma_{5}} \lambda \gamma_{1} c_{2} c_{3} \gamma_{3}^{2} h^{* 2}+17 \gamma_{1} \gamma_{3}^{2} c_{3} h^{*}+O\left(h^{*}\right)+O\left(h^{\frac{1}{2}}\right),
\end{aligned}
$$

where $c_{3}$ is defined by $(3.7)$ and

$$
c_{5}=\max _{i, j \in \mathcal{S}}\left\|\left(L_{i}-L_{j}\right)^{T} R_{i} L_{j}\right\|, \quad c_{6}=\max _{i, j \in \mathcal{S}}\left\|L_{i}^{T} R_{j} L_{i}\right\|
$$

Similar to equation (2.29) of [25], with regard to the system (1.1) and the SD-based adaptive control (2.4) we have

$$
\begin{aligned}
\widetilde{\mathcal{A}}\left(x_{t}^{T} M\left(\theta_{t}\right) x_{t}\right) \triangleq & \lim _{\Delta \rightarrow 0} \frac{1}{\Delta}\left(E\left[x_{t+\Delta}^{T} M\left(\theta_{t+\Delta}\right) x_{t+\Delta} \mid \theta_{t}\right]-x_{t}^{T} M\left(\theta_{t}\right) x_{t}\right) \\
= & x_{t}^{T}\left(A^{T}\left(\theta_{t}\right) M\left(\theta_{t}\right)+M\left(\theta_{t}\right) A\left(\theta_{t}\right)+\sum_{j} \lambda_{\theta_{t}, j} M_{j}\right) x_{t}+\operatorname{tr}\left(C^{T}\left(\theta_{t}\right) M\left(\theta_{t}\right) C\left(\theta_{t}\right)\right) \\
& +u_{t}^{T} B^{T}\left(\theta_{t}\right) M\left(\theta_{t}\right) x_{t}+x_{t}^{T} M\left(\theta_{t}\right) B\left(\theta_{t}\right) u_{t},
\end{aligned}
$$


where $\widetilde{\mathcal{A}}$ is the infinitesimal operator of associated process $\left\{\theta_{t}, x_{t}\right\}$. Hence, by Dynkin formula [22] we have

$$
\begin{aligned}
E\left[x_{t}^{T} M\left(\theta_{t}\right) x_{t}\right]= & x_{0}^{T} M\left(\theta_{0}\right) x_{0}+E \int_{0}^{t} \operatorname{tr}\left(C^{T}\left(\theta_{s}\right) M\left(\theta_{s}\right) C\left(\theta_{s}\right)\right) \mathrm{d} s \\
& +E \int_{0}^{t}\left(u_{s}^{T} B^{T}\left(\theta_{s}\right) M\left(\theta_{s}\right) x_{s}+x_{s}^{T} M\left(\theta_{s}\right) B\left(\theta_{s}\right) u_{s}\right) \mathrm{d} s \\
& +E \int_{0}^{t} x_{s}^{T}\left(A^{T}\left(\theta_{s}\right) M\left(\theta_{s}\right)+M\left(\theta_{s}\right) A\left(\theta_{s}\right)+\sum_{j} \lambda_{\theta_{s}, j} M_{j}\right) x_{s} \mathrm{~d} s .
\end{aligned}
$$

Associating Riccati equation (2.2) with the above equation, we get

$$
\begin{aligned}
E\left[x_{t}^{T} M\left(\theta_{t}\right) x_{t}+\int_{0}^{t}\left(x_{s}^{T} Q\left(\theta_{s}\right) x_{s}+u_{s}^{T} R\left(\theta_{s}\right) u_{s}\right) \mathrm{d} s\right]= & x_{0}^{T} M\left(\theta_{0}\right) x_{0}+E \int_{0}^{t} \operatorname{tr}\left(C^{T}\left(\theta_{s}\right) M\left(\theta_{s}\right) C\left(\theta_{s}\right)\right) \mathrm{d} s \\
& +E \int_{0}^{t}\left(u_{s}-u_{s}^{*}\right)^{T} R\left(\theta_{s}\right)\left(u_{s}-u_{s}^{*}\right) \mathrm{d} s .
\end{aligned}
$$

Thus, by (4.24) we have

$$
\begin{aligned}
\limsup _{t \rightarrow \infty} E\left(\frac{1}{t} x_{t}^{T} M\left(\theta_{t}\right) x_{t}+\frac{1}{t} \int_{0}^{t}\left(x_{s}^{T} Q\left(\theta_{s}\right) x_{s}+u_{s}^{T} R\left(\theta_{s}\right) u_{s}\right) \mathrm{d} s\right)= & \limsup _{t \rightarrow \infty} \frac{1}{t} E \int_{0}^{t} \operatorname{tr}\left(C^{T}\left(\theta_{s}\right) M\left(\theta_{s}\right) C\left(\theta_{s}\right)\right) \mathrm{d} s \\
& +\limsup _{t \rightarrow \infty} \frac{1}{t} E \int_{0}^{t}\left(u_{s}-u_{s}^{*}\right)^{T} R\left(\theta_{s}\right)\left(u_{s}-u_{s}^{*}\right) \mathrm{d} s \\
\leq & \limsup _{t \rightarrow \infty} \frac{1}{t} E \int_{0}^{t} \operatorname{tr}\left(C^{T}\left(\theta_{s}\right) M\left(\theta_{s}\right) C\left(\theta_{s}\right)\right) \mathrm{d} s \\
& +\frac{340}{\gamma_{5}} \lambda \gamma_{1} c_{2} c_{3} \gamma_{3}^{2} h^{* 2}+17 \gamma_{1} c_{3} \gamma_{3}^{2} h^{*} \\
& +O\left(h^{*}\right)+O\left(h^{\frac{1}{2}}\right),
\end{aligned}
$$

which derives (3.4), by the definitions of $\gamma_{3}$ and $\gamma_{5}$ (see (4.20) and the line below (4.23), respectively). Thus, Theorem 3.1 is true.

Remark 4.4. By the proof and the conclusion of Theorem 3.1, the influence of system noise on the performance index is crucial. As control step goes to zero, the difference between the performance index under the SD-based LQ control and the optimal performance index under the complete-state-information-based LQ control does not approach to zero, but to some constant

$$
\frac{340 \lambda \gamma_{1} c_{2} c_{3}\left(\frac{2}{\gamma_{2}}+1\right)^{2} h^{* 2}}{1-10 c_{2} h^{*}\left(2 \lambda+\lambda_{1} h^{*}\right)-\frac{2 c_{2} \gamma_{0}}{1-\gamma_{0}}}+17 \gamma_{1} c_{2}\left(\frac{2}{\gamma_{2}}+1\right)^{2} h^{*}+O\left(h^{*}\right),
$$

which is determined by the system parameter set and estimation step $h^{*}$. It is worth noticing that the key error term is $17 \gamma_{1} c_{2}\left(\frac{2}{\gamma_{2}}+1\right)^{2} h^{*}$, which has the order of $h^{*-1}$ as $h^{*}$ decreases to zero. So, in order to reduce the upper bound (3.4), we propose to choose a relatively large estimation step $h^{*}$ within some admissible range, and a sufficiently small control step $h$ for control updating. (3.2) is only a sufficient condition. And so, for a concrete system, the estimation step $h^{*}$ may be selected to be larger than that given by (3.2). 


\section{Simulation EXAmple}

In this section, we will give a simulation example to show the influence of estimation step $h^{*}$ and control step $h$ on the LQ performance index.

Consider one dimension MJS (1.1)-(1.3) with

$$
N=2 ; A_{1}=0 ; A_{2}=-1 ; B_{1}=1 ; B_{2}=1 ; C_{1}=1 ; C_{2}=1 ; \Lambda=\left[\begin{array}{cc}
-1 & 1 \\
0 & 0
\end{array}\right] .
$$

Let $R_{1}=10 ; R_{2}=0.1 ; Q_{1}=1 ; Q_{2}=1$ in LQ performance index (1.4).

Obviously, for $i=1,2,\left[A_{i}, Q_{i}^{\frac{1}{2}}\right]$ is observable. By [12], $\left[A\left(\theta_{t}\right), B\left(\theta_{t}\right)\right]$ is stochastically stabilizable. The unique symmetric positive definite solution set of coupled algebra Riccati equations (2.2) is

$$
M_{1}=1.1091, \quad M_{2}=0.232 .
$$

Feedback control matrices are

$$
L_{1}=0.11091, \quad L_{2}=2.32 .
$$

The solutions of algebra Riccati equations (4.11) are

$$
K_{1}=0.942, \quad K_{2}=0.151 .
$$

First, we consider one-step approach to design SD-based adaptive control. In this case, the estimation step is equal to the control step, that is, $h^{*}=h$. Two different values $h=0.005$ and $h=0.0005$ are taken, respectively. The index difference between the performance index $J$ under SD-based LQ control and the optimal performance index $\limsup _{t \rightarrow \infty} \frac{1}{t} E \int_{0}^{t} \operatorname{tr}\left(C^{T}\left(\theta_{s}\right) M\left(\theta_{s}\right) C\left(\theta_{s}\right)\right) \mathrm{d} s$ under the complete-state-information-based LQ control can be expressed as

$$
\Delta J=J-\limsup _{t \rightarrow \infty} \frac{1}{t} E \int_{0}^{t} \operatorname{tr}\left(C^{T}\left(\theta_{s}\right) M\left(\theta_{s}\right) C\left(\theta_{s}\right)\right) \mathrm{d} s,
$$

and depicted in Figures 1 and 2, respectively.

From Figures 1 and 2 it can be seen that when $h^{*}=h=0.005$, the index difference $\Delta J$ is about 6 at the end of our simulation; but when $h^{*}=h=0.0005, \Delta J$ is finally about 7 . This tells us that by one-step approach, smaller sample step does not mean better performance index.

To get a clearer illustration about this observation, we run the simulation 25 times under $h^{*}=h=0.005$ and $h^{*}=h=0.0005$, respectively. As the existence of random uncertainty caused by Brownian motion and Markov jumping in system (1.1), from each simulation we get quite inequable index differences. To describe system index difference in an average way, also a reasonable way, we list the index differences $\Delta J_{i}$ as well as the mean index differences $\overline{\Delta J}=\frac{1}{25} \sum_{i=1}^{25} \Delta J_{i}$ in Tables 1 and 2. By simple calculation, in the first case $\left(h^{*}=h=0.005\right)$, the mean index difference $\overline{\Delta J}=4.1744$; when sample step reduces to $h^{*}=h=0.0005, \overline{\Delta J}=4.3822$. Obviously, the performance index is neither asymptotically convergent to the optimal value nor decreasing smaller as $h$ reduces. Actually, the performance index under the SD-based adaptive LQ control seems worse as the sample step becomes smaller in the case of the one-step approach.

Since the one-step approach cannot ensure good system performance, by Theorem 3.1 we employ the DSapproach to design SD-based adaptive control for system (5.1) so as to optimize the LQ performance index. For system (5.1), one can easily verify that distinguishable condition (3.1) holds for any given $h^{*}>0$. Noticing that too large estimation step $h^{*}$ is not good for parameter estimation, and hence, not favorable to the system performance, condition (3.3) given in Theorem 3.1 restricts the range of $h^{*}$. By straightforward calculation, all $h^{*}$ in $[0.0012,0.0018]$ satisfy condition (3.3). In this example, the range of $h^{*}$ is indeed wider than $[0.0012,0.0018]$, which is consistent with the discussion of Remark 3.4. Actually, we can choose, for example, $h^{*}=0.05$ and $h^{*}=0.005$, respectively, in the following double-stepped simulation. 

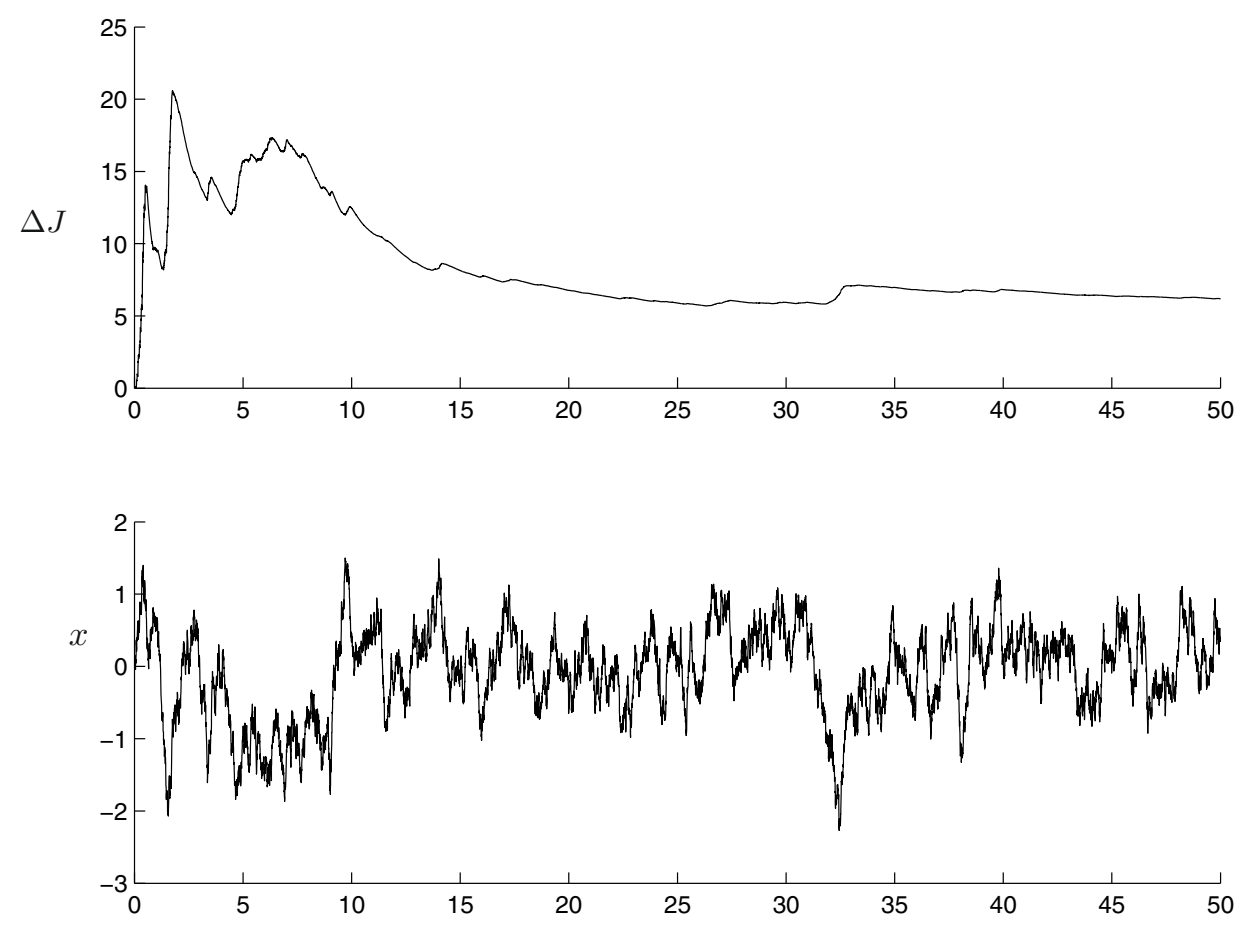

Figure 1. Curves of $\Delta J, x$, when $h^{*}=h=0.005$.

To illustrate how greatly the DS-approach improves the system performance and how much the estimation step $h^{*}$ and control step $h$ influence $\Delta J$, we run the simulation 25 times for each of the following three groups: (1) $h^{*}=0.05, h=0.001 ;(2) h^{*}=0.005, h=0.001$; and $(3) h^{*}=0.05, h=0.01$. The differences $\Delta J_{i}$ and the mean differences $\overline{\Delta J}$ are listed in Tables $3-5$, respectively.

According to the simulation results listed in Tables 1, 3 and 4, we have the following comparison about the one-step approach and DS-approach:

- By the one-step approach, when $h^{*}=h=0.005$, the mean difference $\overline{\Delta J}=4.1744$. However, by the DS-approach, when estimation step is kept at $h^{*}=0.005$ and the control step is reduced to $h=0.001$, the mean difference becomes 3.3068, which is obviously better than that obtained by one-step approach.

- By the DS-approach with estimation step $h^{*}=0.05$ and control step $h=0.001, \overline{\Delta J}$ equals 0.0993 , much better than that obtained by the one-step approach.

Based on the above analysis, DS-approach is obviously much better than the one-step approach in terms of system performance.

Relying on those simulation results listed in Tables $3-5$, we have the following observations about the impact of the DS- and the one-step approach on the system performance:

- With respect to the fixed control step $h=0.001$, when estimation steps are taken as $h^{*}=0.05$ and $h^{*}=0.005$, respectively, the corresponding index differences $\overline{\Delta J}$ are 0.0993 and 3.3068, respectively. This indicates that for a given control step, too small estimation step may worsen the performance index.

- With respect to the fixed estimation step $h^{*}=0.05$, when control steps are taken as $h=0.001$ and $h=0.01$, respectively, the corresponding differences $\overline{\Delta J}$ are 0.0993 and 2.4464 , respectively. This indicates that for a given estimation step, smaller control step may enhance the system performance. 

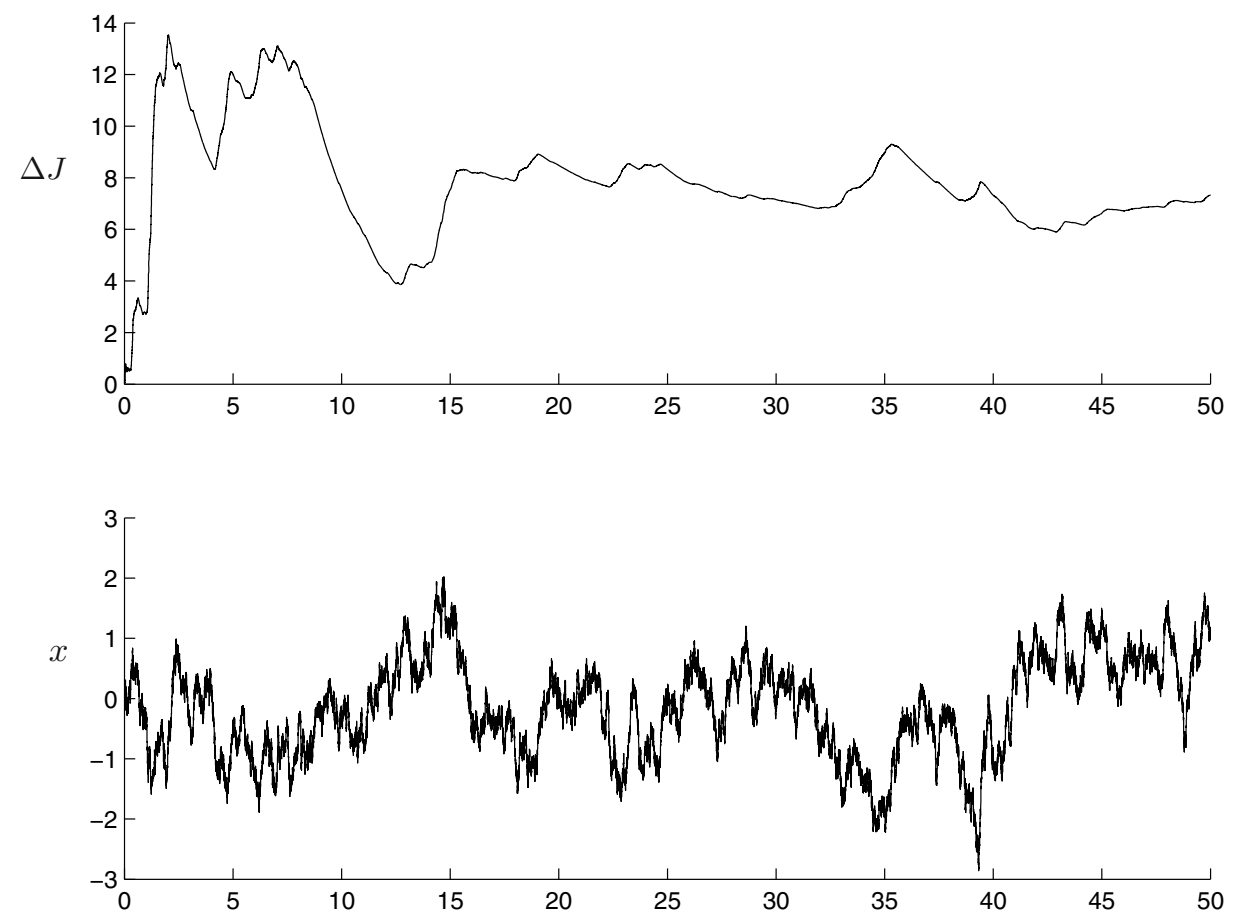

Figure 2. Curves of $\Delta J, x$, when $h^{*}=h=0.0005$.

TABLE 1. Values of $\Delta J_{i}$ and $\overline{\Delta J}$, when $h^{*}=h=0.005$.

\begin{tabular}{|c|c|c|c|c|c|}
\hline \multirow{4}{*}{$\Delta J$} & 9.2479 & 7.3104 & 7.5697 & 0.0881 & 5.6875 \\
& 9.7960 & -0.1188 & 0.0155 & 8.8376 & 8.1260 \\
& 7.9942 & 8.4289 & 4.7325 & -0.1184 & -0.0943 \\
& 8.8710 & -0.0834 & -0.0780 & 5.0272 & 4.5475 \\
& 5.2946 & -0.0355 & -0.1266 & 7.6809 & 0.0374 \\
\hline \multicolumn{6}{|c|}{$\overline{\Delta J}=4.1744$} \\
\hline
\end{tabular}

- From the third group $h^{*}=0.05, h=0.01$, we see that when estimation step and control step are reduced (by the same percentage $90 \%$ ) to $h^{*}=0.005, h=0.001$, respectively, the index difference $\overline{\Delta J}$ increases from 2.4464 to 3.3068 , and becomes worse. This together with the above two observations indicates that reduction of estimation step may be the key factor that worsens the system performance.

In summary, the key factor influencing system performance is estimation step rather than control step. So, when designing SD-based adaptive control by DS-approach, in order to get a good performance index, a smart way is to choose a suitable estimation step $h^{*}$ and a sufficiently small control step $h$.

\section{Conclusion}

In this paper we study the SD-based adaptive LQ control problem of a class of MJS with unknown Markov jumps and stochastic noises, and analyze the influence of sample step on both the stability and the performance index of the closed-loop system. In order to reduce the co-influence of Markov jumps and stochastic noises on parameter estimates, system states and performance index, we introduce a DS-approach to design adaptive control. This DS-approach is characterized by two different sample steps. One is much larger than the other. 
TABLE 2. Values of $\Delta J_{i}$ and $\overline{\Delta J}$, when $h^{*}=h=0.0005$.

\begin{tabular}{|c|c|c|c|c|c|}
\hline \multirow{4}{*}{$\Delta J$} & 8.0151 & 9.3887 & 7.0258 & 5.2822 & -0.1919 \\
& 0.3630 & 7.2570 & 9.3738 & 5.1325 & 5.4405 \\
& 6.0733 & 0.1194 & -0.0929 & 7.2268 & 10.3951 \\
& 0.0400 & 6.3659 & 7.9925 & -0.1279 & 0.0466 \\
& -0.0251 & 6.4008 & 0.5221 & -0.0815 & 7.6143 \\
\hline \multicolumn{5}{|c|}{$\overline{\Delta J}=4.3822$} \\
\hline
\end{tabular}

TABLE 3. Values of $\Delta J_{i}$ and $\overline{\Delta J}$, when $h^{*}=0.05$ and $h=0.001$.

\begin{tabular}{|c|c|c|c|c|c|}
\hline \multirow{4}{*}{$\Delta J$} & -4.2595 & 2.3691 & 1.3762 & -0.2489 & -1.4393 \\
& -1.8598 & 1.9844 & 0.1006 & -0.3818 & -0.7565 \\
& -0.3711 & 0.8186 & 0.2877 & -0.4120 & -0.2960 \\
& -0.7688 & 3.7420 & -1.9723 & -0.1786 & 0.0542 \\
& 0.6156 & 0.9287 & -0.1997 & 3.5687 & -0.2186 \\
\hline \multicolumn{5}{|c|}{$\overline{\Delta J}=0.0993$} \\
\hline
\end{tabular}

TABLE 4 . Values of $\Delta J_{i}$ and $\overline{\Delta J}$, when $h^{*}=0.005$ and $h=0.001$.

\begin{tabular}{|c|c|c|c|c|c|}
\hline \multirow{4}{*}{$\Delta J$} & 7.3655 & 5.8146 & -0.0360 & -0.1370 & 5.2980 \\
& 8.3120 & 4.0067 & 7.5286 & 3.2389 & 12.5092 \\
& -0.1294 & -0.0169 & -0.5097 & 4.2209 & -0.2194 \\
& 9.1987 & 5.7351 & -0.1010 & 7.0010 & -0.1715 \\
& 0.0153 & -0.0480 & 4.1415 & -0.1313 & -0.2147 \\
\hline \multicolumn{6}{|c|}{$\overline{\Delta J}=3.3068$} \\
\hline
\end{tabular}

TABLE 5. Values of $\Delta J_{i}$ and $\overline{\Delta J}$, when $h^{*}=0.05$ and $h=0.01$.

\begin{tabular}{|c|c|c|c|c|c|}
\hline \multirow{4}{*}{$\Delta J$} & -0.2313 & 7.4997 & -3.9841 & -0.2458 & 6.2383 \\
& 5.7968 & 8.3235 & -0.0484 & 0.0060 & 0.5342 \\
& -0.1112 & 3.3286 & 5.1799 & 2.7506 & 3.7324 \\
& -1.7149 & -0.0931 & -0.2892 & 2.8978 & -0.1414 \\
& 2.9570 & 10.0084 & -0.1719 & 7.4188 & 1.5205 \\
\hline \multicolumn{6}{|c|}{$\overline{\Delta J}=2.4464$} \\
\hline
\end{tabular}

The larger one is used to estimate the unknown Markov jumps so as to reduce the influence of the stochastic noise on the estimation accuracy and that of the system noise and estimation errors on the performance index. The smaller one is used to design the SD-based control, since the smaller the control step is, the more information on the system states can be obtained, and so, better controller can be obtained. With the assumptions that MJS is stochastically stabilizable and each subsystem is observable, we prove that the closed-loop system is stable under the double-stepped SD-based adaptive LQ control. It is found that in some circumstances, too small estimation step may worsen the system performance, although for the control step, the smaller the better.

\section{Appendix A: Proofs of Lemmas 4.2-4.4}

Proof of Lemma 4.2. Since almost all sample paths $\left\{\theta_{t}\right\}$ are constant except for a finite number of simple jumps in any finite time interval [25], assume that the jump points in $\left[s_{0}-h, s_{0}\right]$ are $m$, and denote them by $s_{1}, s_{2}$, $\ldots, s_{m}$ in sequence:

$$
s_{0}-h<s_{1}<s_{2}<\ldots<s_{m}<s_{m+1}=s_{0} .
$$


Then, from

$$
E\left[\left\|g\left(\theta_{s^{\prime}}\right)-g\left(\hat{\theta}_{s_{0}}\right)\right\| \int_{s^{\prime}}^{s} f\left(\theta_{\mu}\right) \mathrm{d} \mu I_{\mathcal{G}_{s_{0}-h}} \mid \theta_{s_{0}-h}=i_{0}\right] \leq\left(s-s^{\prime}\right) \max _{i, j \in \mathcal{S}}\|g(i)-g(j)\| \max _{k \in \mathcal{S}} f(k) E I_{\mathcal{G}_{s_{0}-h}}
$$

and

$$
\begin{gathered}
\sum_{j \neq i_{0}} P_{i_{0} j}(h) \leq h \sum_{j \neq i_{0}}\left(\frac{\mathrm{e}^{\Lambda h}-I}{h}\right)_{i_{0} j} \leq h \sum_{j \neq i_{0}}\left\|\frac{\mathrm{e}^{\Lambda h}-I}{h}\right\| \leq \lambda h, \\
P_{i_{0} i_{0}}(h)-\prod_{i=0}^{m} P_{i_{0} i_{0}}\left(s_{i+1}-s_{i}\right) \leq P_{i_{0} i_{0}}(h)-\left(1+\lambda_{i_{0} i_{0}} h\right)=h^{2}\left(\frac{\mathrm{e}^{\Lambda h}-I-\Lambda h}{h^{2}}\right)_{i_{0} i_{0}} \leq \lambda_{1} h^{2},
\end{gathered}
$$

one can get Lemma 4.2.

Proof of Lemma 4.3. Let

$$
F_{i j}=\mathrm{e}^{A_{i} h}-\int_{0}^{h} \mathrm{e}^{A_{i}(h-\tau)} \mathrm{d} \tau B_{i} L_{j}
$$

Then, spirited by the Taylor expressions:

$$
\mathrm{e}^{x}=\sum_{s=0}^{\infty} \frac{x^{s}}{s !}, \quad \log (1+x)=\sum_{n=1}^{\infty} \frac{(-1)^{n+1} x^{n}}{n}(|x|<1), \quad \ln \frac{1}{1-x}=\sum_{n=1}^{\infty} \frac{x^{n}}{n}(|x|<1),
$$

from condition (3.3) we have

$$
\begin{aligned}
& F_{i j}-I=\sum_{s=0}^{\infty} \frac{A_{i}^{s} h^{s+1}}{(s+1) !}\left(A_{i}-B_{i} L_{j}\right), \\
& \left\|F_{i j}-I\right\| \leq h\left\|A_{i}-B_{i} L_{j}\right\|\left\|\sum_{s=0}^{\infty} \frac{A_{i}^{s} h^{s}}{(s+1) !}\right\| \leq d_{1}<1, \\
& \log F_{i j}=\sum_{n=1}^{\infty} \frac{(-1)^{n+1}\left(F_{i j}-I\right)^{n}}{n} \\
& \left\|\log F_{i j}\right\| \leq \sum_{n=1}^{\infty} \frac{\left\|F_{i j}-I\right\|^{n}}{n} \leq \ln \frac{1}{1-d_{1}}, \\
& \left\|F_{i j}^{N^{*}}\right\|=\left\|\mathrm{e}^{N^{*} \log F_{i j}}\right\| \leq \mathrm{e}^{N^{*} \ln \frac{1}{1-d_{1}}}=\left(1-d_{1}\right)^{N^{*}}<1, \\
& \sum_{s=1}^{\infty} \frac{\left\|A_{i}\right\|^{s} h^{s}}{s !} \leq \mathrm{e}^{c_{1} h}-1
\end{aligned}
$$

where $d_{1}$ is given by (4.7).

By (A.1) and (A.3) we have

$$
\begin{aligned}
\log \left(F_{i j}^{N^{*}}\right) & =N^{*}\left[\left(A_{i}-B_{i} L_{j}\right) h+\sum_{s=1}^{\infty} \frac{A_{i}^{s} h^{s+1}}{(s+1) !}\left(A_{i}-B_{i} L_{j}\right)+\sum_{n=2}^{\infty} \frac{(-1)^{n+1}\left(F_{i j}-I\right)^{n}}{n}\right] \\
& =\left(A_{i}-B_{i} L_{j}\right) h^{*}+\Delta_{i j}
\end{aligned}
$$

where

$$
\Delta_{i j}=h^{*} \sum_{s=1}^{\infty} \frac{A_{i}^{s} h^{s}}{(s+1) !}\left(A_{i}-B_{i} L_{j}\right)+N^{*} \sum_{n=2}^{\infty} \frac{(-1)^{n+1}\left(F_{i j}-I\right)^{n}}{n}
$$


By (A.2), (A.4) and (A.6) we have

$$
\begin{aligned}
\left\|\Delta_{i j}\right\| & =\left\|h^{*} \sum_{s=1}^{\infty} \frac{A_{i}^{s} h^{s}}{(s+1) !}\left(A_{i}-B_{i} L_{j}\right)+N^{*}\left(F_{i j}-I\right)^{2} \sum_{n=0}^{\infty} \frac{(-1)^{n+1}\left(F_{i j}-I\right)^{n}}{n+2}\right\| \\
& \leq h^{*}\left\|A_{i}-B_{i} L_{j}\right\| \sum_{s=1}^{\infty} \frac{\left\|A_{i}\right\|^{s} h^{s}}{s !}+N^{*} d_{1}^{2}\left(\frac{1}{2}+\sum_{n=1}^{\infty} \frac{\left\|F_{i j}-I\right\|^{n}}{n}\right) \\
& <2 c_{1} h^{*}\left(\mathrm{e}^{c_{1} h}-1\right)+4 c_{1}^{2} h^{*} h \mathrm{e}^{2 c_{1} h}\left(\frac{1}{2}+\ln \frac{1}{1-d_{1}}\right)
\end{aligned}
$$

For any given matrices $A$ and $B$, similar to Example x.4.2 in [2], one can get

$$
\mathrm{e}^{A+B}=\mathrm{e}^{A}+\int_{0}^{1} \mathrm{e}^{(1-t) A} B \mathrm{e}^{t(A+B)} \mathrm{d} t .
$$

This together with (A.7) leads to

$$
\begin{aligned}
\mathrm{e}^{\left(A_{k}-B_{k} L_{j}\right) h^{*}}+\mathrm{e}^{\left(A_{i}-B_{i} L_{j}\right) h^{*}}-2 F_{i j}^{N^{*}}= & \mathrm{e}^{\left(A_{k}-B_{k} L_{j}\right) h^{*}}+\mathrm{e}^{\left(A_{i}-B_{i} L_{j}\right) h^{*}}-2\left(\mathrm{e}^{\left(A_{i}-B_{i} L_{j}\right) h^{*}}\right. \\
& \left.+\int_{0}^{1} \mathrm{e}^{(1-t)\left(A_{i}-B_{i} L_{j}\right) h^{*}} \Delta_{i j} F_{i j}^{t N^{*}} \mathrm{~d} t\right) \\
= & \mathrm{e}^{\left(A_{k}-B_{k} L_{j}\right) h^{*}}-\mathrm{e}^{\left(A_{i}-B_{i} L_{j}\right) h^{*}}-2 \int_{0}^{1} \mathrm{e}^{(1-t)\left(A_{i}-B_{i} L_{j}\right) h^{*}} \Delta_{i j} F_{i j}^{t N^{*}} \mathrm{~d} t \\
= & \mathcal{D}_{k j i}\left(h^{*}\right)\left(I-2 \mathcal{D}_{k j i}^{-1}\left(h^{*}\right) \int_{0}^{1} \mathrm{e}^{(1-t)\left(A_{i}-B_{i} L_{j}\right) h^{*}} \Delta_{i j} F_{i j}^{t N^{*}} \mathrm{~d} t\right)
\end{aligned}
$$

Hence, from (3.3), (A.5) and (A.8) we arrive at

$$
\begin{aligned}
\left\|2 \mathcal{D}_{k j i}^{-1}\left(h^{*}\right) \int_{0}^{1} \mathrm{e}^{(1-t)\left(A_{i}-B_{i} L_{j}\right) h^{*}} \Delta_{i j} F_{i j}^{t N^{*}} \mathrm{~d} t\right\| & \leq 2\left\|\mathcal{D}_{k j i}^{-1}\left(h^{*}\right)\right\|\left\|\Delta_{i j}\right\| \int_{0}^{1}\left\|\mathrm{e}^{(1-t)\left(A_{i}-B_{i} L_{j}\right) h^{*}}\right\|\left\|F_{i j}\right\|^{t N^{*}} \mathrm{~d} t \\
& <2\left\|\mathcal{D}_{k j i}^{-1}\left(h^{*}\right)\right\| 4 c_{1}^{2} h^{* 2} \mathrm{e}^{2 c_{1} h^{*}}\left[\left(\mathrm{e}^{c_{1} h}-1\right)\right. \\
& \left.+2 c_{1} h \mathrm{e}^{2 c_{1} h}\left(\frac{1}{2}+\ln \frac{1}{1-d_{1}}\right)\right] \\
& <1
\end{aligned}
$$

which implies that the matrix $I-2 \mathcal{D}_{k j i}^{-1}\left(h^{*}\right) \int_{0}^{1} \mathrm{e}^{(1-t)\left(A_{i}-B_{i} L_{j}\right) h^{*}} \Delta_{i j} F_{i j}^{t N^{*}} \mathrm{~d} t$ is nonsingular. Hence, by the nonsingularity of $\mathcal{D}_{k j i}\left(h^{*}\right)$ and (A.9), the matrix $\mathrm{e}^{\left(A_{k}-B_{k} L_{j}\right) h^{*}}+\mathrm{e}^{\left(A_{i}-B_{i} L_{j}\right) h^{*}}-2 F_{i j}^{N^{*}}$ is nonsingular. This renders $\gamma_{2}>0$.

Proof of Lemma 4.4. For any given integers $k=0,1, \ldots, l=0,1, \ldots, N^{*}-1$, and real number $t \in\left[k h^{*}+\right.$ $\left.l h, k h^{*}+l h+h\right)$, substituting the SD-based adaptive control (2.4) into the system (1.1), one can get the following closed-loop system

$$
\begin{aligned}
\mathrm{d} x_{t} & =A\left(\theta_{t}\right) x_{t} \mathrm{~d} t-B\left(\theta_{t}\right) L\left(\hat{\theta}_{k h^{*}}\right) x_{t^{\prime}} \mathrm{d} t+C\left(\theta_{t}\right) \mathrm{d} W_{t} \\
& =A\left(\theta_{t}\right)\left(x_{t}-x_{k h^{*}}\right) \mathrm{d} t+A\left(\theta_{t}\right) x_{k h^{*}} \mathrm{~d} t-B\left(\theta_{t}\right) L\left(\hat{\theta}_{k h^{*}}\right) x_{t^{\prime}} \mathrm{d} t+C\left(\theta_{t}\right) \mathrm{d} W_{t} .
\end{aligned}
$$


Let

$$
\begin{aligned}
\theta_{(k-1) h^{*}} & =i, \quad \hat{\theta}_{(k-1) h^{*}}=j, \quad \hat{\theta}_{k h^{*}}=k, \\
\overline{\mathcal{G}}_{(k-1) h^{*}} & =\left\{\omega: \omega \in \Omega, \theta_{t}(\omega) \text { has no jump on interval }\left[(k-1) h^{*}, k h^{*}\right)\right\},
\end{aligned}
$$

and notice that the sample path of $x$ is continuous with probability one. Then, by (A.11) we obtain

$$
x_{k h^{*}}=F_{i j}^{N^{*}} x_{(k-1) h^{*}}+\widetilde{W}_{k h^{*}} \text { a.s., }
$$

where

$$
\begin{aligned}
& F_{i j}=\mathrm{e}^{A_{i} h}-\int_{0}^{h} \mathrm{e}^{A_{i}(h-\tau)} \mathrm{d} \tau B_{i} L_{j}, \\
& \widetilde{W}_{k h^{*}}=\sum_{r=0}^{N^{*}-1} F_{i j}^{r} \widetilde{W}_{k h^{*}-(r+1) h}, \\
& \widetilde{W}_{k h^{*}-(r+1) h}=\int_{k h^{*}-(r+1) h}^{k h^{*}-r h} \mathrm{e}^{A_{i}\left(k h^{*}-r h-s\right)} C_{i} \mathrm{~d} W_{s} .
\end{aligned}
$$

Since stochastic variables $\left\{\widetilde{W}_{k h^{*}-(r+1) h}, r=0, \ldots, N^{*}-1\right\}$ and Markov process $\left\{\theta_{t}\right\}$ are mutually independent, by (A.5) we have

$$
E\left\|\widetilde{W}_{k h^{*}}\right\|^{2} \leq \sum_{r=0}^{N^{*}-1} E\left\|F_{i j}^{r}\right\|^{2} E\left\|\widetilde{W}_{k h^{*}-(r+1) h}\right\|^{2} \leq N^{*} E \int_{0}^{h} \operatorname{tr}\left(C_{i}^{T} \mathrm{e}^{A_{i}^{T} s} \mathrm{e}^{A_{i} s} C_{i}\right) \mathrm{d} s .
$$

From the LMEE (2.3) it follows that

$$
\left\|F_{i j}^{N^{*}} x_{(k-1) h^{*}}-\mathrm{e}^{\left(A_{k}-B_{k} L_{j}\right) h^{*}} x_{(k-1) h^{*}}+\widetilde{W}_{k h^{*}}\right\| \leq\left\|F_{i j}^{N^{*}} x_{(k-1) h^{*}}-\mathrm{e}^{\left(A_{i}-B_{i} L_{j}\right) h^{*}} x_{(k-1) h^{*}}+\widetilde{W}_{k h^{*}}\right\| .
$$

Squaring both sides of the above inequality and removing the same terms from the both sides gives

$$
\left\|\left(\mathrm{e}^{\left(A_{k}-B_{k} L_{j}\right) h^{*}}+\mathrm{e}^{\left(A_{i}-B_{i} L_{j}\right) h^{*}}-2 F_{i j}^{N^{*}}\right) x_{(k-1) h^{*}}\right\| \leq 2\left\|\widetilde{W}_{k h^{*}}\right\| .
$$

This together with Lemma 4.3 implies

$$
\left\|x_{(k-1) h^{*}}\right\| \leq \frac{2}{\gamma_{2}}\left\|\widetilde{W}_{k h^{*}}\right\|
$$

Thus, by (A.5) and (A.13) we have

$$
\left\|x_{k h^{*}}\right\|=\left\|F_{i j}^{N^{*}} x_{(k-1) h^{*}}+\widetilde{W}_{k h^{*}}\right\| \leq\left\|F_{i j}^{N^{*}}\right\|\left\|x_{(k-1) h^{*}}\right\|+\left\|\widetilde{W}_{k h^{*}}\right\| \leq \gamma_{3}\left\|\widetilde{W}_{k h^{*}}\right\| .
$$

Integrating both sides of (A.12) on interval $\left[k h^{*}, k h^{*}+l h\right)$ leads to

$$
\begin{aligned}
x_{\left(k h^{*}+l h\right)}-x_{k h^{*}}= & \int_{k h^{*}}^{k h^{*}+l h} A\left(\theta_{t}\right)\left(x_{t}-x_{k h^{*}}\right) \mathrm{d} t+\int_{k h^{*}}^{k h^{*}+l h} A\left(\theta_{t}\right) \mathrm{d} t x_{k h^{*}}+\int_{k h^{*}}^{k h^{*}+l h} C\left(\theta_{t}\right) \mathrm{d} W_{t} \\
& -\sum_{i=0}^{l-1} \int_{k h^{*}+i h}^{k h^{*}+(i+1) h} B\left(\theta_{t}\right) \mathrm{d} t L\left(\hat{\theta}_{k h^{*}}\right) x_{\left(k h^{*}+i h\right)},
\end{aligned}
$$


which implies

$$
\begin{aligned}
\left\|x_{\left(k h^{*}+l h\right)}-x_{k h^{*}}\right\| \leq & c_{1} \int_{k h^{*}}^{k h^{*}+l h}\left\|x_{t}-x_{k h^{*}}\right\| \mathrm{d} t+c_{1} h^{*}\left\|x_{k h^{*}}\right\|+c_{1} h \sum_{i=0}^{l-1}\left\|x_{k h^{*}+i h}\right\| \\
& +\left\|\int_{k h^{*}}^{k h^{*}+l h} C\left(\theta_{t}\right) \mathrm{d} W_{t}\right\| .
\end{aligned}
$$

This together with the Gronwall inequality renders

$$
\begin{aligned}
\left\|x_{\left(k h^{*}+l h\right)}-x_{k h^{*}}\right\| \leq & \mathrm{e}^{c_{1} h^{*}}\left[c_{1} h^{*}\left\|x_{k h^{*}}\right\|+c_{1} h \sum_{i=0}^{l-1}\left\|x_{\left(k h^{*}+i h\right)}\right\|\right]+\left\|\int_{k h^{*}}^{k h^{*}+l h} C\left(\theta_{t}\right) \mathrm{d} W_{t}\right\| \\
& +c_{1} \mathrm{e}^{c_{1} h^{*}} \int_{k h^{*}}^{k h^{*}+l h}\left\|\int_{k h^{*}}^{s} C\left(\theta_{\mu}\right) \mathrm{d} W_{\mu}\right\| \mathrm{d} s,
\end{aligned}
$$

or equivalently,

$$
\begin{aligned}
\left\|x_{\left(k h^{*}+l h\right)}\right\| \leq & \left(1+c_{1} h^{*} \mathrm{e}^{c_{1} h^{*}}\right)\left\|x_{k h^{*}}\right\|+c_{1} h \mathrm{e}^{c_{1} h^{*}} \sum_{i=0}^{l-1}\left\|x_{\left(k h^{*}+i h\right)}\right\|+\left\|\int_{k h^{*}}^{k h^{*}+l h} C\left(\theta_{t}\right) \mathrm{d} W_{t}\right\| \\
& +c_{1} \mathrm{e}^{c_{1} h^{*}} \int_{k h^{*}}^{k h^{*}+l h}\left\|\int_{k h^{*}}^{s} C\left(\theta_{\mu}\right) \mathrm{d} W_{\mu}\right\| \mathrm{d} s .
\end{aligned}
$$

By (A.16), (A.15) and Lemma 4.3 we arrive at

$$
\begin{aligned}
\left\|x_{\left(k h^{*}+l h\right)}\right\| \leq & \left(1+d_{3}\right)\left(1+\mathrm{e}^{c_{1} h^{*}} c_{1} h\right)^{l-1}\left\|x_{k h^{*}}\right\|+\sum_{i=1}^{l-1}\left[\sum_{j=1}^{l-i}\left(\mathrm{e}^{c_{1} h^{*}} c_{1} h\right)^{j} d\left(k h^{*}+i h\right)\right] \\
& +d\left(k h^{*}+l h\right) \\
\leq & \left(1+d_{3}\right)\left(1+\mathrm{e}^{c_{1} h^{*}} c_{1} h\right)^{l-1} \gamma_{3}\left\|\widetilde{W}_{k h^{*}}\right\|+\sum_{i=1}^{l-1}\left[\sum_{j=1}^{l-i}\left(\mathrm{e}^{c_{1} h^{*}} c_{1} h\right)^{j} d\left(k h^{*}+i h\right)\right] \\
& +d\left(k h^{*}+l h\right),
\end{aligned}
$$

and hence,

$$
\begin{aligned}
\left\|x_{\left(k h^{*}+l h\right)}\right\|^{2} \leq & 2\left(1+d_{3}\right)^{2}\left(1+\mathrm{e}^{c_{1} h^{*}} c_{1} h\right)^{2 l-2} \gamma_{3}^{2}\left\|\widetilde{W}_{k h^{*}}\right\|^{2}+4 d^{2}\left(k h^{*}+l h\right) \\
& +4\left[\sum_{i=1}^{l-1} \sum_{j=1}^{l-i}\left(\mathrm{e}^{c_{1} h^{*}} c_{1} h\right)^{j} d\left(k h^{*}+i h\right)\right]^{2},
\end{aligned}
$$

where

$$
\begin{aligned}
& d_{3}=c_{1} h^{*} \mathrm{e}^{c_{1} h^{*}}+c_{1} h \mathrm{e}^{c_{1} h^{*}}, \\
& d\left(k h^{*}+i h\right)=c_{1} \mathrm{e}^{c_{1} h^{*}} \int_{k h^{*}}^{k h^{*}+i h}\left\|\int_{k h^{*}}^{s} C\left(\theta_{\mu}\right) \mathrm{d} W_{\mu}\right\| \mathrm{d} s+\left\|\int_{k h^{*}}^{k h^{*}+i h} C\left(\theta_{t}\right) \mathrm{d} W_{t}\right\| .
\end{aligned}
$$


Notice that condition (3.2) ensures $c_{1} h \mathrm{e}^{c_{1} h^{*}}<1$. Then, by Schwarz inequality, the last term on the right hand of (A.18) satisfies

$$
\begin{aligned}
4\left[\sum_{i=1}^{l-1} \sum_{j=1}^{l-i}\left(\mathrm{e}^{c_{1} h^{*}} c_{1} h\right)^{j} d\left(k h^{*}+i h\right)\right]^{2} & \leq 4 \sum_{i=1}^{l-1}\left[\sum_{j=1}^{l-i}\left(\mathrm{e}^{c_{1} h^{*}} c_{1} h\right)^{j}\right]^{2} \sum_{i=1}^{l-1} d^{2}\left(k h^{*}+i h\right) \\
& \leq \frac{4 l \mathrm{e}^{2 c_{1} h^{*}} c_{1}^{2} h^{2}}{\left(1-\mathrm{e}^{c_{1} h^{*}} c_{1} h\right)^{2}} \sum_{i=1}^{l-1} d^{2}\left(k h^{*}+i h\right) .
\end{aligned}
$$

By Schwarz inequality and the Fubini theorem [17], we have

$$
\begin{aligned}
E d^{2}\left(k h^{*}+i h\right) & \leq 2 E\left\|\int_{k h^{*}}^{k h^{*}+i h} C\left(\theta_{s}\right) \mathrm{d} W s\right\|^{2}+2 h^{*} c_{1}^{2} \mathrm{e}^{2 c_{1} h^{*}} \int_{k h^{*}}^{k h^{*}+i h} E\left\|\int_{k h^{*}}^{s} C\left(\theta_{\mu}\right) \mathrm{d} W \mu\right\|^{2} \mathrm{~d} s \\
& =2 \int_{k h^{*}}^{k h^{*}+i h} \operatorname{tr}\left(C^{T}\left(\theta_{s}\right) C\left(\theta_{s}\right)\right) \mathrm{d} s+2 h^{*} c_{1}^{2} \mathrm{e}^{2 c_{1} h^{*}} \int_{k h^{*}}^{k h^{*}+i h} \int_{k h^{*}}^{s} \operatorname{tr}\left(C^{T}\left(\theta_{\mu}\right) C\left(\theta_{\mu}\right)\right) \mathrm{d} \mu \mathrm{d} s \\
& \leq 2 c_{4} i h+c_{4} c_{1}^{2} \mathrm{e}^{2 c_{1} h} h^{*} h^{2} i^{2}
\end{aligned}
$$

integrating both sides of (A.18) on time interval $\left[k h^{*}+l h, k h^{*}+(l+1) h\right)$, taking expectation, summing over all the $K N^{*}$ terms, by (A.14), (A.19) and (A.20) one can get

$$
\begin{aligned}
& \sum_{k=0}^{K-1} \sum_{l=1}^{N^{*}-1} E \int_{k h^{*}+l h}^{k h^{*}+(l+1) h}\left\|x_{\left(k h^{*}+l h\right)}\right\|^{2} \mathrm{~d} s \leq 2\left(1+d_{3}\right)^{2} \gamma_{3}^{2} \sum_{k=0}^{K-1} \sum_{l=1}^{N^{*}-1} h\left(1+\mathrm{e}^{c_{1} h^{*}} c_{1} h\right)^{2 l-2} E\left\|\widetilde{W}_{k h^{*}}\right\|^{2} \\
&+4 \sum_{k=0}^{K-1} \sum_{l=1}^{N^{*}-1} h E d^{2}\left(k h^{*}+l h\right)+\frac{4 \mathrm{e}^{2 c_{1} h^{*}} c_{1}^{2}}{\left(1-\mathrm{e}^{c_{1} h^{*}} c_{1} h\right)^{2}} \sum_{k=0}^{K-1} \sum_{l=1}^{N^{*}-1} l \sum_{i=1}^{l} h^{3} E d^{2}\left(k h^{*}+i h\right) \\
& \leq 2\left(1+d_{3}\right)^{2} \gamma_{3}^{2} K h^{* 2} \mathrm{e}^{2 c_{1} h^{*} \mathrm{e}^{c_{1} h^{*}}} \frac{1}{h} E \int_{0}^{h} \operatorname{tr}\left(C_{i}^{T} \mathrm{e}^{A_{i}^{T} s} \mathrm{e}^{A_{i} s} C_{i}\right) \mathrm{d} s \\
&+\left[\frac{4 \mathrm{e}^{e c_{2} h^{*}} c_{1}^{2} h^{* 2}}{\left(1-\mathrm{e}^{c_{1} h^{*}} c_{1} h\right)^{2}}+4\right] K h^{* 2}\left(c_{4}+c_{4} c_{1}^{2} \mathrm{e}^{2 c_{1} h} h^{*}\right),
\end{aligned}
$$

where the following inequalities have been used for the last inequality:

$$
\begin{gathered}
\sum_{l=1}^{N^{*}-1}\left(1+c_{1} h \mathrm{e}^{c_{1} h^{*}}\right)^{2 l-2}=\frac{\left(1+c_{1} h \mathrm{e}^{c_{1} h^{*}}\right)^{2 N^{*}-2}-1}{\left(1+c_{1} h \mathrm{e}^{c_{1} h^{*}}\right)^{2}-1}<\frac{\mathrm{e}^{2 c_{1} h^{*} \mathrm{e}^{c_{1} h^{*}}}-1}{2 c_{1} h \mathrm{e}^{c_{1} h^{*}}}<\frac{h^{*}}{h} \mathrm{e}^{2 c_{1} h^{*} \mathrm{e}^{c_{1} h^{*}}}, \\
\sum_{l=1}^{N^{*}-1} h E d^{2}\left(k h^{*}+l h\right) \leq c_{4} h^{* 2}+c_{4} c_{1}^{2} \mathrm{e}^{2 c_{1} h} h^{* 4} \\
\sum_{l=1}^{N^{*}-1} l \sum_{i=1}^{l-1} h^{3} E d^{2}\left(k h^{*}+i h\right) \leq h^{3} \sum_{l=1}^{N^{*}-1} l \sum_{i=1}^{l-1}\left(2 c_{4} i h+c_{4} c_{1}^{2} \mathrm{e}^{2 c_{1} h}(i h)^{2}\right) \\
\leq h^{3} \sum_{l=1}^{N^{*}-1}\left(c_{4} h l^{3}+c_{4} c_{1}^{2} \mathrm{e}^{2 c_{1} h} h^{2} l^{4}\right) \leq c_{4} h^{* 4}+c_{4} c_{1}^{2} \mathrm{e}^{2 c_{1} h} h^{* 6}
\end{gathered}
$$

Noticing condition (3.2) implies $c_{1} h^{*} \mathrm{e}^{c_{1} h^{*}} \leq \frac{\sqrt{2}-1}{2}$, by (A.21) we have Lemma 4.4. 


\section{REFERENCES}

[1] K.J. Åström and B. Wittenmark, Computer-Controlled Systems: Theory and Design. Third edition, Tsinghua University Press (2002).

[2] R. Bhatia, Matrix Analysis. Springer Verlag (1996).

[3] P.E. Caines and J.F. Zhang, On the adaptive control of jump parameter systems via nonlinear filtering. SIAM J. Contr. Opt. 33 (1995) $1758-1777$.

[4] Y.S. Chow and H. Teicher, Probability Theory: Independence, Interchangeability, Martingales. Springer Verlag (1978).

[5] E.A. Coddington and R. Carlson, Linear Ordinary Differential Equations. SIAM (1997).

[6] F. Dufour and P. Betrand, Stabilizing control law for hybrid models. IEEE Trans. Automat. Contr. 39 (1994) $2354-2357$.

[7] R.J. Elliott and V. Krishnamurthy, Exact finite dimensional filters for maximum likelihood parameter estimation of continuous time linear-Gaussian systems. SIAM. J. Contr. Opt. 35 (1997) 1908-1923.

[8] R.J. Elliott and V. Krishnamurthy, New finite dimensional filters for parameter estimation of discrete time linear Gaussian models. IEEE. Trans. Automat. Contr. 44 (1999) 938-951.

[9] L.S. Hu, Y.-Y. Cao and H.-H. Shao, Constrained robust sampled-data control for nonlinear uncertain systems. Int. J. Robust Nonlinear Contr. 12 (2002) 447-464.

[10] M.Y. Huang and L. Guo, Stabilization of stochastic systems with hidden Markov jumps. Science in China (Series F) 44 (2001) $104-118$.

[11] A. Ilchmann and S. Townley, Adaptive sampling control of high-gain stabilizable systems. IEEE Trans. Automat. Contr. 44 (1999) 1961-1966.

[12] Y.D. Ji and H.J. Chizeck, Controllability, stabilizability and continuous-time Markovian jump linear quadratic control. IEEE Trans. Automat. Contr. 35 (1990) 777-788.

[13] Y.D. Ji and H.J. Chizeck, Jump linear quadratic Guassian control in continuous time. IEEE Trans. Automat. Contr. 37 (1992) $1884-1892$.

[14] A. Kanchanaharuthai, Optimal sampled-data controller design with time-multiplied performance index for load-frequency control, in Proceedings of the 2004 IEEE international conference on control applications (2004) 655-660.

[15] G. Kreisselmeier and R. Lozano, Adaptive control of continuous-time overmodeled plants. IEEE Trans. Automat. Contr. 41 (1996) 1779-1794.

[16] H.J. Kushner, Stochastic Stability and Control. Academic Press (1967).

[17] R.S. Lipster and A.N. Shiryaev, Statistics of Random Processes I. General Theory. Second edition, Springer Verlag (2001).

[18] M. Mariton, Jump Linear Systems in Automatic Control. Marcel Dekker Inc. (1990).

[19] O. Ocah and M.E. Sezer, Robust adaptive sampled-data control of a class of systems under structured nonlinear perturbations. IEEE Trans. Automat. Contr. 42 (1997) 553-558.

[20] Y. Oishi, A bound of conservativeness in sampled-data robust stabilization and its dependence on sampling periods. Systems Control Lett. 32 (1997) 11-19.

[21] R. Ortega and G. Kreisselmeier, Discrete-time model reference adaptive control for continuous-time systems using generalized sampled-Data hold functions. IEEE Trans. Automat. Contr. 35 (1990) 334-338.

[22] P. Protter, Stochastic Integration and Differential Equations: A New Approach. Springer-Verlag (1990).

[23] D.D. Sworder, Hybrid adaptive control. Appl. Math. Comput. 45 (1991) 173-192.

[24] S.P. Tan, J.-F. Zhang and L.L. Yao, Optimality analysis of adaptive sampled control of hybrid systems with quadratic index. IEEE Trans. Automat. Contr. 50 (2005) 1044-1051.

[25] W.M. Wonham, Random Differential Equations in Control Theory, in Probabilistic Methods in Applied Mathematics 2 , A.T. Bharucha-Reid Ed., Academic Press (1970).

[26] F. Xue and L. Guo, Necessary and sufficient conditions for adaptive stability of jump linear systems. Communications in Information and Systems $\mathbf{1}$ (2001) 205-224.

[27] L.L. Yao and J.F. Zhang, Sampled-data-based LQ control of stochastic linear continuous-time systems. Science in China (Series F) 45 (2002) 383-396.

[28] R. Yu, O. Ocali and M.E. Sezer, Adaptive robust sampled-data control of a class of systems under structured perturbations. IEEE Trans. Automat. Contr. 38 (1993) 1707-1713.

[29] C. Zhang, R.H. Middleton and R.J. Evans, An algorithm for multirate sampling adaptive control. IEEE Trans. Automat. Contr. 34 (1989) 792-795. 